\title{
Dynamic Preference for Flexibility
}

\author{
R. Vijay Krishna \\ UNC Chapel Hill
}

\author{
Philipp Sadowski
}

Duke University

\author{
July 19, 2012
}

ERID Working Paper Number 133

This paper can be downloaded without charge from the Social Science Research Network Electronic Paper Collection:

http://ssrn.com/abstract=2149771

\section{Economic Research Initiatives at Duke WORKING PAPERS SERIES

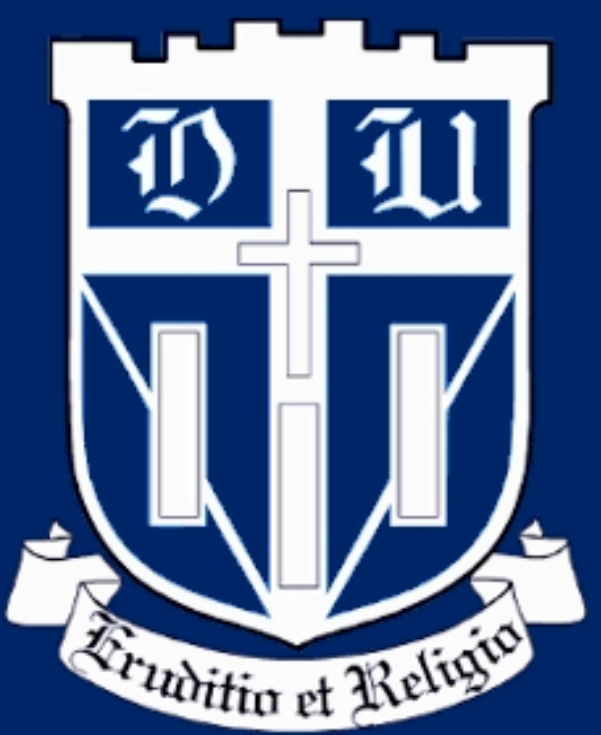




\title{
Dynamic Preference for Flexibility*
}

\author{
R. VIJAY KrishNA ${ }^{\dagger} \quad$ PhILIPP SADOWSKI \\ ThurSDAY $19^{\mathrm{TH}}$ JULY, 2012
}

\begin{abstract}
We consider a decision maker who experiences transient preference shocks when faced with dynamic decision situations that involve intertemporal tradeoffs, such as those in consumption savings problems. We axiomatize a recursive representation of choice over infinite horizon consumption problems that features uncertain consumption utilities that evolve according to a subjective process that is iid. A generalization of the model introduces objective states of the world and accommodates persistent taste shocks that are transient, contingent on the state. In the corresponding representation the uncertainty about utilities depends on the exogenous state, which follows a subjective Markov process. The parameters of the representations, which are the subjective processes governing the evolution of beliefs over consumption utilities, and the discount factor, are uniquely identified from behavior. We characterize a natural notion of greater preference for flexibility in terms of a dilation of beliefs.
\end{abstract}

\section{Introduction}

Uncertainty about future consumption utilities influences how economic agents make decisions. A decision maker (DM) who is uncertain about future consumption utilities, perhaps due to uncertain future risk aversion or other taste shocks, prefers not to commit to a course of future action today and therefore has preference for flexibility. For example, DM might be willing to forfeit current consumption if this allows him to delay a decision about future consumption. While this intuition is

(*) We would like to thank, without implicating, Haluk Ergin, Sujoy Mukerji, Wolfgang Pesendorfer, Todd Sarver, Norio Takeoka, three anonymous referees, and a co-editor for helpful comments and suggestions, and Vivek Bhattarcharya, Matt Horne, and Justin Valasek for valuable research assistance.

(†) University of North Carolina, Chapel Hill <rvk@unc . edu $>$

(\$) Duke University $<$ p. sadowski@duke. edu $>$ 
inherently dynamic, standard models that accommodate preference for flexibility, most prominently Kreps [1979] and Dekel, Lipman and Rustichini] [2001] (henceforth $\mathrm{DLR}^{1}$ ), are static in the sense that there is no intertemporal tradeoff (although Kreps [1979] suggests that an infinite horizon model of preference for flexibility is desirable).

We first focus on transient taste shocks as the source of preference for flexibility. As a generalization, we then consider taste shocks that have an unobservable transient as well as an observable persistent component. We provide foundations for recursive representations that are fully identified.

We follow Gul and Pesendorfer [2004], henceforth GP, and consider infinite horizon consumption problems (IHCPs) as the domain of choice. IHCPs are defined recursively as menus of lotteries over pairs of consumption and new IHCPs as continuation problems. For a simple example of an IHCP that features only degenerate lotteries, suppose DM has income $y$ in every period and current wealth $w$. The price of consumption, $\rho>0$, is fixed. In each period DM can choose to consume an amount $k \in\left[0, \frac{w+y}{\rho}\right]$ at cost $k \rho$. This will leave him with wealth $w^{\prime}=w+y-k \rho$ for the next period. Given this technology, we can formulate the consumption problem he faces recursively as a collection of feasible consumption and continuation problem pairs,

$$
x_{w}:=\left\{\left(k, x_{w^{\prime}}\right): k \in\left[0, \frac{w+y}{\rho}\right], w^{\prime}=w+y-k \rho\right\}
$$

The domain of IHCPs is rich and allows for complicated future choice behavior ${ }^{2}$ For example, future choices may depend directly on time or the consumption history. We rule out such dependencies in order to stay as close to the standard model as possible. ${ }^{3}$ Given two IHCPs $x$ and $y$, a decision maker who does not anticipate any taste shocks is indifferent between $x$ and $x \cup y$ whenever he prefers $x$ to $y$. Following Kreps [1979], we refer to this property as strategic rationality. In contrast, a decision maker who anticipates taste shocks satisfies Monotonicity, that is, he weakly prefers $x \cup y$ to $x$.

Strategic rationality greatly simplifies the analysis of DM's behavior. If strategic rationality with respect to all IHCPs is violated because of unobservable taste shocks, then it is desirable to understand the nature of this violation by asking if DM is strategically rational with respect to some smaller class of IHCPs. In particular, if taste shocks are transient (relevant only for one period), then DM should be strategically rational when restricting next period's choice of a continuation problem. This is the content of our axiom Continuation Strategic Rationality (CSR).

Based on CSR, we provide a representation that is the solution to a Bellman

(1) A relevant corrigendum is Dekel, Lipman, Rustichini and Sarver [2007] (henceforth DLRS).

(2) We only model the initial choice of a consumption problem, but our representation suggests dynamically consistent future choice.

(3) Dependence of choice on the consumption history is central to the notion of habits. We leave a model of habit formation on the domain of IHCPs as a topic for future research. 
equation, and can therefore be analyzed using standard dynamic programming techniques. It features uncertain consumption utilities that evolve according to a subjective iid process. All the parameters, which are the discount factor and the probability measure over consumption utilities in each period, are uniquely identified.

In order to accommodate observable persistent shocks, we introduce exogenous states of the world that may evolve over time, and that can be contracted upon. If all the persistence of the taste shocks is captured by the state, then taste shocks are transient contingent on the state. Consequently, DM should be strategically rational with respect to continuation problems, contingent on the state. This is our axiom State Contingent CSR. In the resulting model the evolution of uncertainty about future consumption utilities is driven by the evolution of the (exogenous) state of the world, which in turn follows a subjective Markov process. Again, all the parameters of the representation are uniquely identified. In the special case where, contingent on the state, there is not even preference for flexibility with respect to consumption alternatives, the model reduces to a fully identified recursive version of the Anscombe-Aumann model with state dependent utilities.

A supplement, Krishna and Sadowski [2012b], provides foundations for a fully identified model that accommodates preference for flexibility due to unobservable persistent taste shocks.

Our representations are generalizations of 'taste shock' models that are commonly used in applied work. Our axioms spell out the testable implications of such models. For example, if the model features iid transient taste shocks, then choice should satisfy CSR. To test this assumption, consider two assets that pay the same iid dividend in each period. The only difference is that the first asset cannot be resold in the future, while the second asset can be re-sold in the next period. An agent who satisfies CSR should be willing to pay the same initial price for both assets. If, on the contrary, the observed price of the second asset is greater than the price of the first asset in a statistically significant way, then CSR is violated, and hence the iid model is misspecified. One possible explanation for such a violation could be that transient taste shocks depend on the state of the economy. In that case choice should satisfy State Contingent CSR, which can be tested in a similar fashion.

Our identification results allow us to characterize a notion of greater preference for flexibility in terms of a dilation (which is the multi-dimensional generalization of a mean preserving spread) of beliefs over consumption utilities. Based on this characterization, Krishna and Sadowski [2012a] analyze how prices in a particular Lucas tree economy react to a dilation of the beliefs of a representative agent who has dynamic preference for flexibility.

Identification is also relevant for model based forecasting, which is a central reason for the use of formal models. It involves estimating a relevant parameter in one context in order to forecast outcomes in another. Model based forecasting, thus, requires that (i) the relevant parameter is uniquely identified and (ii) the modeler is 
willing to assume that the parameter is meaningful outside the context of the observed data. In our models beliefs over consumption utilities are uniquely identified. Those beliefs might, for example, forecast future choice frequencies of alternatives from continuation problems, as discussed in Sadowski [2012].

The remainder of the paper is structured as follows. Section 2 reviews related literature. Section 3 lays out the basic framework without objective states. Based on CSR, it derives a recursive representation where DM's utilities follow a subjective iid process. Section 4 considers an environment with objective states and preferences that satisfy State Contingent CSR, and derives a representation where beliefs about consumption utilities depend on the exogenous state, and states follow a subjective Markov process. A characterization of greater preference for flexibility can be found in section 5 while section 6 concludes. All proofs, as well as a general representation of preference for flexibility with an infinite prize space, are established in the appendices.

\section{Related Literature}

Our work builds on standard axiomatic models of preference for flexibility. The innovation that makes it possible to think about preference for flexibility, introduced by Kreps [1979], is the investigation of choice over menus of consumption outcomes. The second seminal paper in this literature, DLR, modifies the domain to consider menus of lotteries over outcomes as objects of choice. This facilitates the interpretation of the subjective states as tastes, where a taste is simply a (twice normalized) vN-M ranking over consumption outcomes. This is a distinct advantage over Kreps since the space of all twice normalized vN-M functions is a well behaved and analytically tractable set. While menu choice can capture DM's attitude towards the future, implied choice in these models is actually static, in the sense that there is no opportunity for any intertemporal tradeoff. We provide a model of preference for flexibility due to uncertain consumption utilities in the tradition of these earlier papers, where the implied choice is dynamic.

We adopt the recursive domain of Infinite Horizon Consumption Problems (IHCPs), first analyzed by GP, who show that this recursive domain is well defined. GP provide a dynamic model of consumption with temptation preferences. We allow, instead, for uncertain utilities on the domain of IHCPs. In principle, this requires an infinite dimensional subjective state space, which complicates the analysis. Higashi, Hyogo and Takeoka [2009], henceforth HHT, use the same domain and also consider preference for flexibility. They provide a stationary representation, where the preference for flexibility stems exclusively from a random discount factor. This elegantly avoids the issue of the dimensionality of the state space, but the model cannot capture uncertainty about future consumption utilities such as uncertain risk aversion, and does not accommodate non-trivial evolution of uncertainty. Takeoka 
[2006] considers choice between menus of menus of lotteries, and derives a three period version of DLR. The model cannot capture intertemporal tradeoffs, and consequently the representation is only jointly identified, as in DLR. Rustichini [2002] is also interested in preference for flexibility in intertemporal problems, modeled as lotteries over sets of infinite consumption paths. While an additive representation can be obtained, the domain precludes a recursive value function and does not allow unique identification of the representation.

Static models of preference for flexibility have difficulty uniquely identifying parameters from behavior, as beliefs and utilities cannot be distinguished. DLR suggest the introduction of a numeraire good (with state independent evaluation) to their model, so as to identify beliefs uniquely in the same sense that they are uniquely identified in Anscombe and Aumann [1963]. The source of identification in the PFC representation is similar, where the numeraire arises naturally in the form of continuation problems. 5 Sadowski [2012] considers a situation where observable states of the world contain some information that is 'relevant' for future preferences and shows that beliefs over subjective states can then be identified. Instead of choice between menus, Gul and Pesendorefer [2006] study random choice from menus. Observed choice frequencies naturally correspond to a unique measure over utilities, but the scaling of utilities remains arbitrary in their model. Ahn and Sarver [2012] simultaneously model choice between menus and random choice from menus. They achieve full identification by requiring the beliefs in the representation of choice between menus to correspond to frequencies of choice from menus. Dillenberger and Sadowski [2012] and Takeoka [2007] fully identify representations of preference for flexibility which feature uncertain future beliefs, rather than uncertain future tastes.

That a decision maker can be uncertain about future preferences in a dynamic setting is noted by Koopmans [1964], who distinguishes between once-and-for-all planning, where the agent selects an action for all possible future contingencies, and piecemeal planning where, in each period, the agent chooses an action for the current period, while simultaneously narrowing the set of alternatives for the future. Jones and Ostroy [1984] consider a non-axiomatic (but dynamic) model of choice where an agent prefers flexibility due to uncertainty about future utilities. They point out that preference for flexibility, for example in the form of preference for liquidity, is a pervasive theme in economics, and relate many instances in macroeconomics and finance where DM's dynamic behavior exhibits preference for liquidity in particular, and preference for flexibility in general, as in Goldman [1974]. They also discuss a notion of greater variability of beliefs which roughly corresponds to our notion of a dilation of beliefs. A special case of the PFC representation is used in empirical work by Hendel and Nevo [2006], who study the problem of a decision maker who

(4) Schenone [2010] formalizes this argument.

(5) In contrast, continuation utilities in the PFX representation do not provide a numeraire, as they are state dependent. Instead, identification follows directly from the recursive structure of the representation. 
maintains an inventory.

A growing literature argues that variations in preferences over time, and in particular in risk-aversion, are central in explaining various market behaviors. Indeed, estimated risk aversion has been suggested as a useful index of market sentiment and there is evidence that the largest component of changes of the equity risk premium is variation in risk aversion, rather than the quantity of risk..$^{7}$ More generally, variation of risk aversion over time has been considered in representative agent settings to improve our understanding of asset pricing phenomena. For instance, Campbell and Cochrane [1999] identify variations in risk premia that correlate with the fundamentals of the economy as a crucial aspect of many dynamic asset pricing phenomena $\sqrt{8}$ Bekaert et al [2010] show that allowing stochastic risk aversion that is not driven by, or perfectly correlated with, the fundamentals of the economy can explain a wide range of asset pricing phenomena, as well as fitting important features of bond and stock markets simultaneously. Our PFX representation, where the evolution of utilities can depend on the state of the world, can accommodate both cases.

As mentioned above, an immediate application of our model is provided in Krishna and Sadowski [2012a], who investigate a Lucas tree economy with an investment stage and a representative agent whose preferences have a PFC representation. They show that greater variation in risk aversion results in greater price volatility, and also in underinvestment, since investing in the productive asset reduces liquidity.

\section{Unobserved Transient Shocks}

In this section we describe the environment and provide a model of preference for flexibility with transient shocks.

\subsection{Environment}

For a compact metric space $Y$, let $\mathscr{P}(Y)$ denote the space of probability measures endowed with the topology of weak convergence, so that $\mathscr{P}(Y)$ is compact and metrizable. Let $\mathscr{F}(Y)$ denote the space of closed subsets of a compact metric space $Y$, endowed with the Hausdorff metric, which makes $\mathscr{F}(Y)$ a compact metric space.

Let $K$ be a finite set of consumption prizes with typical member $k$. We follow GP in defining an infinite horizon consumption problem (IHCP) as a collection of

(6) See, for example, Bollerslev et al [2011].

(7) See Smith and Whitelaw [2009].

(8) In Campbell and Cochrane [1999] the correlation is generated indirectly, via habit forming consumption choices. Empirically, however, it is the correlation itself that is important and not the particular mechanism (such as habit formation) that drives it, as Bekaert et al] [2010] point out. 
lotteries that yield a prize in the present period and a new infinite horizon problem starting in the next period. Let $Z$ be the collection of all IHCPs. $9^{9}$ GP show that $Z$ is a compact metric space, and that each $z \in Z$ can be identified with a compact set of probability measures over $K \times Z$. In particular, it can be shown that $Z$ is linearly homeomorphic to the space of all closed subsets of $\mathscr{P}(K \times Z)$. We shall denote this linear homeomorphism as $Z \simeq \mathscr{F}(\mathscr{P}(K \times Z))$. Typical elements $x, y, z \in Z$ are interpreted as menus of lotteries over consumption and continuation problems, while $p, q \in \mathscr{P}(K \times Z)$ are typical lotteries, with $p_{k}$ and $p_{z}$ denoting the marginal distributions of $p$ on $K$ and $Z$.

We explicitly model choice between consumption problems from an ex-ante perspective, before consumption begins. That is, we analyze a binary relation $\succsim$ $\subset Z \times Z$, which we refer to as a preference. We let $\succ$ and $\sim$ denote, respectively, the asymmetric and symmetric parts of $\succsim$. The recursive domain of IHCPs is rich; for instance, it can accommodate temporal lotteries as in Kreps and Porteus [1978]. It is also amenable to analysis by stochastic dynamic programming. Its construction follows the descriptive approach of Kreps and Porteus [1978] in that it more closely describes how economic agents act and, as mentioned above, embodies what Koopmans [1964] refers to as piecemeal planning: instead of choosing a consumption stream that determines consumption for all time, at each instant the decision maker chooses immediate consumption as well as a set of alternatives for the future.

We will also consider the space of menus of consumption lotteries, $\mathscr{F}(\mathscr{P}(K))$, with typical members being $a, b, c$. By the recursive nature of $Z$, continuation problems are members of $Z$. Let $A, B, C$ denote typical elements of the collection of menus of continuation lotteries, $\mathscr{F}(\mathscr{P}(Z))$. To ease notational burden, we will often write $\mathscr{F}$ for $\mathscr{F}(\mathscr{P}(K \times Z)), \mathscr{F}_{K}$ for $\mathscr{F}(\mathscr{P}(K))$, and $\mathscr{F}_{Z}$ for $\mathscr{F}(\mathscr{P}(Z))$. When there is no risk of confusion, we identify prizes and continuation problems with degenerate lotteries and lotteries with singleton menus. For example, we denote the lottery over continuation problems that yields $z$ with certainty by $z$, and the lottery that yields current consumption $k$ and continuation problem $x$ with certainty by $(k, x)$.

\subsection{Axioms}

Our first two axioms on $\succsim$ collect standard requirements.

Aхıом 1 (Non-triviality). There exist $x, y \in Z$ such that $x \succ y$.

Aхıом 2 (Continuous Order). $\succsim$ satisfies the following:

(a) $\succsim$ is complete and transitive.

(b) $\succsim$ is continuous, in the sense that $\{y: y \succsim x\}$ and $\{y: x \succsim y\}$ are closed.

(9) See GP for the recursive construction of $Z$. We provide a sketch in appendix C 
We take the convex sum of sets to be the Minkowski sum, namely $\lambda x+(1-$ ג) $y:=\{\lambda p+(1-\lambda) q: p \in x, q \in y\}$ whenever $\lambda \in[0,1]$. Notice that if $x, y \in \mathscr{F}$, then $\lambda x+(1-\lambda) y$ is also closed, and hence is in $\mathscr{F}$. The following axiom is the usual Independence axiom.

Axıом 3 (Independence). $x \succ y$ implies $\lambda x+(1-\lambda) z \succ \lambda y+(1-\lambda) z$ for all $\lambda \in(0,1]$ and $z \in Z \mathbb{1 0}$

We are interested in a DM who anticipates preference shocks, and hence values flexibility.

Aхıом 4 (Monotonicity). $x \cup y \succsim x$ for all $x, y \in Z$.

This is the central axiom in Kreps [1979]. It says that additional alternatives are always weakly beneficial.

Theorem 4 in appendix $\mathrm{B}$ provides a first representation result for preferences that satisfy Monotonicity (Axiom 4). It establishes that Axioms 14 are necessary and sufficient to afford $\succsim$ a finitely additive $\boldsymbol{E} U$ representation. In particular, there exists a subjective state space, $\mathfrak{U}_{K \times Z}$, which is a collection of all the (twicenormalized) vN-M utility functions on $K \times Z$, along with the Borel algebra $\mathcal{A}_{\mathfrak{U}_{K \times Z}}$, and a charge $\mu$ on $\mathfrak{U}_{K \times Z}$ that induces the preference functional

$$
V(x):=\int_{\mathfrak{U}_{K \times Z}} \max _{p \in x} \mathfrak{u}(p) \mathrm{d} \mu(\mathfrak{u})
$$

The state space $\mathfrak{U}_{K \times Z}$ consists of all continuous functions on $K \times Z$ that are identified up to positive affine transformation. In particular, all the utility functions in $\mathfrak{U}_{K \times Z}$ have (i) the same utility for some $x^{*} \in Z$, and (ii) the same (supremum) norm. The first requirement corresponds to normalizing the constant term to 0 , and the second requirement amounts to normalizing the scaling factor to 1,11

We remark that even though $K$ is finite, $Z$ is infinite and infinite dimensional. This implies we cannot appeal to the additive EU representation theorem of DLR. The representation above is an infinite dimensional version of their representation. There, the state space and the charge over the subjective state space are jointly identified in the sense that given the normalization of the state space, the charge is unique. In contrast, Theorem 4 does not establish joint identification of the finitely additive EU representation. The following axioms leverage the recursive nature of the domain to impose more structure on the vN-M utility functions $\mathfrak{t} \in \mathfrak{U}_{K \times Z}$.

(10) A lottery $p \in \mathscr{P}(K \times Z)$ is a singleton menu. A weaker version of Independence is Singleton Independence, which says that Independence holds for all singleton menus. GP show that Singleton Independence along with Stationarity (Axiom 6) and Singleton Indifference to Timing (Axiom 7) imply Independence (Axiom 3) assumed here.

(11) Formally, there exists a unique element $x^{*} \in Z$ such that $x^{*} \simeq\left(p_{k}^{*}, x^{*}\right) \in \mathscr{F}$, where $x^{*}$ consists of the uniform lottery over $K$, namely $p_{k}^{*} \in \mathscr{P}(K)$, in each period. Analogous to the definition in DLRS, we formally have $\mathfrak{U}_{K \times Z}:=\left\{\mathfrak{i t} \in C(K \times Z):\|\mathfrak{u}\|_{\infty}=1, \sum_{k \in K} \mathfrak{t}\left(k, x^{*}\right)=0\right\}$, where $C(K \times Z)$ is the Banach space of continuous functions on $K \times Z$ with the supremum norm. 
Each probability measure $p$ over $K \times Z$ induces marginal distributions $p_{k}$ and $p_{z}$ over $K$ and $Z$ respectively. The next axiom says that DM does not care about correlations between outcomes in $K$ and $Z$, but only cares about the marginal distributions induced by the lotteries in the menu. In particular, if two lotteries induce the same marginal distributions over $K$ and $Z$, then DM does not value the flexibility of having both lotteries available for choice.

Aхгом 5 (Separability). If, for $p, q \in \mathscr{P}(K \times Z)$, the marginal distributions satisfy $p_{k}=q_{k}$ and $p_{z}=q_{z}$, then $\{p, q\} \sim\{p\}$.

Our version of Separability is stronger than the version of separability assumed by GP. Variants of the axiom also appear in HHT and Schenone [2010]. It is instructive to consider what Separability rules out. Let $x_{1}, x_{2} \in Z$ be IHCPs such that $x_{2} \succ x_{1}$, and let $k_{1}, k_{2} \in K$ be prizes such that $k_{2}$ is more valuable than $k_{1}$ (which is to say, $\left(k_{2}, x\right) \succ\left(k_{1}, x\right)$ for all $\left.x \in Z\right)$. Let $p, q \in \mathscr{P}(K \times Z)$ be such that $p$ is uniform over $\left(k_{2}, x_{1}\right)$ and $\left(k_{1}, x_{2}\right)$, while $q$ is uniform over $\left(k_{1}, x_{1}\right)$ and $\left(k_{2}, x_{2}\right)$. Notice that by construction, $p_{k}=q_{k}$ and $p_{z}=q_{z}$. Nevertheless, $p$ hedges between the present and the future, while $q$ does not, and so it does not seem unreasonable for DM to rank $\{p\} \succ\{q\}$. Separability rules this out by insisting that only the respective marginals matter. We hasten to add that virtually all dynamic models used in applications satisfy separability.

Versions of the next two axioms appear in GP, who provide a more detailed discussion. We are interested in stationary preferences, where the ranking of continuation problems does not depend on time. The recursive nature of the domain allows us to capture this notion via the following axiom, which says that if $x \succsim y$, then $x$ is also better than $y$ as a continuation problem after consumption of $k$. Recall that $(k, x)$ denotes the degenerate lottery that gives $(k, x) \in K \times Z$ with probability one.

Ахгом 6 (Stationarity). $\{(k, x)\} \succsim\{(k, y)\}$ if, and only if, $x \succsim y$.

As mentioned above, the domain of IHCPs is rich enough to describe temporal lotteries. We abstract from any preference for the timing of resolution of uncertainty by imposing the following axiom.

Aхгом 7 (Singleton Indifference to Timing). $\{\lambda(k, x)+(1-\lambda)(k, y)\} \sim\{(k, \lambda x+$ $(1-\lambda) y)\}$ for all $\lambda \in[0,1]$.

The axiom states that DM is indifferent between (i) receiving lottery $\lambda(k, x)+$ $(1-\lambda)(k, y)$, which yields consumption $k$ and determines whether the continuation problem will be $x$ or $y$, (early resolution) and (ii) receiving with certainty consumption $k$ and the continuation menu $\lambda x+(1-\lambda) y$ (late resolution).

The next axiom captures transient taste shocks and is our central new assumption.

Axıом 8 (CSR). $\{(k, x)\} \succsim\{(k, y)\}$ implies $\{(k, x)\} \sim\{(k, x),(k, y)\}$. 
In all the menus compared here, next period's consumption is fixed at $k$. The axiom says that there is no preference for flexibility with respect to continuation problems, given $k$. The axiom does not imply that DM is certain about his consumption utility for periods after next period. It only implies that he does not expect this uncertainty to be resolved prior to next period's choice. This is reasonable if the unobservable utility shocks are indeed transient, that is, relevant for only one period. Notice that the axiom is silent on how DM values the option of retaining the alternatives from both $x$ and $y$ for choice two periods ahead. In fact, if $x \cup y \succ x$, which is consistent with Monotonicity (Axiom 4), then Stationarity (Axiom 6) implies $\{(k, x \cup y)\} \succ\{(k, x)\}$. We interpret this ranking, which is not precluded by Axiom 8, as the manifestation of DM's uncertainty about his consumption utility, two periods ahead.

\subsection{Preference for Flexibility with Constant Beliefs}

In line with the intuition behind Axiom 8 , the relevant subjective state space is the set of all $\mathrm{vN}-\mathrm{M}$ utility functions over $K$ that are identified up to an additive constant, $\mathcal{U}:=\left\{u \in \mathbb{R}^{K}: \sum u_{i}=0\right\}$, endowed with the Borel sigma algebra. Subjective states $u \in \mathcal{U}$ are naturally interpreted as consumption utilities, and the two terms are viewed as synonyms. Similarly, in what follows, all probability measures on subjective state spaces are interpreted as subjective beliefs, and the two terms are used interchangeably. To ensure that expected consumption utility under a measure $\mu$ is well defined, the expected utility from every prize $k \in K$ must be finite. Such a measure $\mu$ is called nice, and so must satisfy $\mu u:=\int_{u} u \mathrm{~d} \mu(u) \in U$.

Definition 1. Let $\mu$ be a nice probability measure on (the Borel sigma-algebra of) $u$, and $\delta \in(0,1)$. We say that $\succsim$ has a representation of Preference for Flexibility with Constant Beliefs (PFC), $(\mu, \delta)$, if there exists a continuous function $V: Z \rightarrow \mathbb{R}$ that satisfies

$$
V(x)=\int_{u} \max _{p \in x}\left[u\left(p_{k}\right)+\delta V\left(p_{z}\right)\right] \mathrm{d} \mu(u)
$$

and represents $\succsim$.

In the representation above, $V$ is linear on $Z$, which simply means $V(\lambda x+$ $(1-\lambda) y)=\lambda V(x)+(1-\lambda) V(y)$ for all $x, y \in Z, \lambda \in[0,1]$. We abuse notation and let $u\left(p_{k}\right)=\sum_{k^{\prime} \in K} p_{k}\left(k^{\prime}\right) u\left(k^{\prime}\right)$ denote the extension of $u$ by linearity. Similarly, $V\left(p_{z}\right)$ denotes the linear extension (by continuity) of $V$ from $Z$ to $\mathscr{P}(Z)$, that is, $V\left(p_{z}\right)=\int_{Z} V(x) \mathrm{d} p_{z}(x)$.

The PFC representation suggests that future utilities over $K$ are uncertain and beliefs follow a subjective process that is iid over time, described by the measure $\mu \cdot 12$

(12) The representation in Hendel and Nevo [2006] is an example of a PFC representation. They 
Proposition 2. Each PFC representation $(\mu, \delta)$ induces a unique continuous function $V \in C(Z)$ that satisfies equation (3.1) above.

The proposition is a corollary of proposition 5 below. In order to identify equivalent PFC representations of $\succsim$, we need the following definition.

Definition 3. Two probability measures $\mu$ and $\mu^{\prime}$ on $U$ are identical up to scaling if there is $\lambda>0$ such that $\mu(D)=\mu^{\prime}(\lambda D)$ for all measurable $D \subset \mathcal{U}$, where $\lambda D:=\{\lambda u: u \in D\}$.

Given a PFC representation $(\mu, \delta)$ of $\succsim$, scaling $\mu$ corresponds to a scaling of the induced value function $V$. Intuitively, for $\lambda=\frac{1}{2}$ all the relevant subjective utilities are twice as large in $\left(\mu^{\prime}, \delta\right)$ as in $(\mu, \delta)$, and hence the induced value function $V^{\prime}$ is also twice as large as $V$.

TheOrem 1. The binary relation $\succsim$ satisfies Axioms $1-8$ if, and only if, it has a PFC representation, $(\mu, \delta)$. Moreover, $\mu$ is unique up to scaling, and $\delta$ is unique.

The theorem is a corollary of Theorem 2 in section 4 below. The uniqueness statement in Theorem 1 is the strongest one could hope for ${ }^{13}$ Let us compare this to the identification obtained in the static model of DLR, where there are no continuation problems and preferences are defined over $\mathscr{F}_{K}$. In DLR, the subjective state space is the collection of all possible consumption rankings - formally, their state space is $\mathfrak{U}_{K}:=\left\{r \in \mathcal{U}: \sum_{i} r_{i}^{2}=1\right\}$ - and preferences are represented by $V(a)=\int_{\mathfrak{U}_{K}} \max _{p_{k} \in a} r\left(p_{k}\right) \mathrm{d} \mu(r)$, where $\mu$ is a probability measure on $\mathfrak{U}_{K}$. The normalization that $\sum_{i} r_{i}^{2}=1$ is arbitrary, and a different normalization - for instance, requiring $\sum_{i}\left|r_{i}\right|^{\tau}=1$ where $\tau \in(1, \infty)$ - would imply a different measure. In that sense, beliefs and utilities are only jointly identified in DLR. In the PFC representation, by contrast, subjective beliefs are uniquely identified (up to scaling) on the state space $u$. Roughly, this is because continuation problems are valued equally in every subjective state, $u \in \mathcal{U}$, which provides a natural numeraire ${ }^{14}$

Since $\mu$ is unique up to scaling, the PFC representation permits the distinction between (stochastic) risk aversion and (stochastic) intertemporal marginal rate of

consider an environment where, in each period, the agent chooses an amount of a good for instantaneous consumption and an amount to add to his inventory. The budget set in each period depends on the realization of prices, which follow a Markov process, and on the inventory. Thus, the choice of inventory constitutes a choice between lotteries over budget sets for next period. This is a version of the IHCP described in the introduction, except that the prices here are random, and hence the lotteries over continuation problems are not degenerate.

(13) The value function $V$ is at best unique up to positive affine transformations. As noted above, scaling the measure $\mu$ corresponds to a scaling of $V$. Adding constants to the vN-M utilities $u(\cdot)$ is also behaviorally meaningless. In the PFC representation, these constants are simply omitted.

(14) As noted in section 1.2, DLR suggest identification of beliefs in a static model of preference for flexibility on the smaller state space $\mathfrak{U}_{K}$ by introducing an artificial numeraire, like money. In the dynamic model the numeraire naturally appears in the form of continuation problems. 
substitution (IMRS). Suppose, for example, that the support of $\mu$ is $\left\{u_{1}, \lambda u_{1}, u_{2}, \lambda u_{2}\right\}$ for some $\lambda>0$. Risk aversion is the same for tastes $u_{i}$ and $\lambda u_{i}$ for $i=1,2$, but the implied IMRS will be different.

In HHT, there is no uncertainty about the ranking of consumption lotteries, only uncertainty about the discount factor. This is a special case of our model where the support of $\mu$ is a subset of $\{u\} \times \mathbb{R}_{+} \subset \mathcal{U}$ for some $u \in U$, with a typical state being $(u, \lambda)$. The utility $u$ represents the ranking, while it is only the intensity $\lambda \in \mathbb{R}_{+}$of the consumption taste that is uncertain. In that case the interpretation of $\delta / \lambda$ as a random discount factor is natural.

\section{Unobserved Transient and Observed Persistent Shocks}

In order to accommodate persistent shocks, we now consider an environment where the objective state of the world may evolve over time, and where DM can make state contingent plans of action. The model we provide allows consumption utilities to correlate with the state of the world.

Many applications involve such objective states that can be contracted upon. Investors can, for example, contract on inflation, dividend growth or other measures of the state of the economy. As we point out in section 2, risk aversion that varies negatively with the state of the economy is essential in explaining a wide range of bond and stock market phenomena.

\subsection{Environment and Axioms}

Let $S:=\{1, \ldots, n\}$ be a finite set of states of the world. For any metric space $Y$, let $\mathscr{H}(Y):=Y^{S}$ denote the space of acts from $S$ to $Y$. In a manner analogous to the approach taken above, we now define a State Contingent Infinite Horizon Consumption Problem (SIHCP) as an act that specifies, for each state of the world, a continuation problem. A continuation problem is a menu of lotteries that yield a consumption prize in the present period and a new SIHCP starting in the next period.

Let $H$ be the collection of all SIHCPs. ${ }^{15} H$ is a compact metric space and is also convex in the sense that for $f, g \in H, \lambda f+(1-\lambda) g \in H$ for all $t \in[0,1]$. Each $f \in H$ can be identified with an act that yields a compact set of probability measures over $K \times H$ in every state. In particular, it can be shown that $H$ is linearly homeomorphic to the space of all acts that take values in $\mathscr{F}(\mathscr{P}(K \times H))$. We shall denote this linear homeomorphism as $H \simeq \mathscr{H}(\mathscr{F}(\mathscr{P}(K \times H)))$, and we will consider a preference relation $\succsim$ on $H$. Typical SIHCPs $f, g \in H$ are acts; typical elements $x, y, z \in \mathscr{F}(\mathscr{P}(K \times H))$ are menus of lotteries over consumption and

(15) We describe the construction of $H$ in appendix C 
SIHCPs; $p, q \in \mathscr{P}(K \times H)$ are typical lotteries; and $p_{k}$ and $p_{h}$ denote the marginal distributions of $p$ on $K$ and $H$ respectively. In what follows, we will abuse notation and refer to the act that gives $x \in \mathscr{F}$ in every state by $x \in H$. Similarly, $(k, f) \in \mathscr{F}$ will refer to the degenerate lottery that yields the sure consumption prize $k \in K$ and the sure SIHCP $f \in H$.

Following the initial choice of an SIHCP, the state of the world, $s \in S$, is realized and DM gets to choose an alternative from the corresponding menu. To define the induced ranking of menus contingent on the state, $\succsim_{s}$, fix $x^{*} \in \mathscr{F}$ and for any $x \in \mathscr{F}$, consider the act

$$
f_{s}^{x}\left(s^{\prime}\right):= \begin{cases}x & \text { if } s=s^{\prime} \\ x^{*} & \text { otherwise }\end{cases}
$$

For any $x, y \in \mathscr{F}$, let $x \succsim_{s} y$ if, and only if, $f_{s}^{x} \succsim_{s}^{y}$. Under our axioms below, $\succsim_{s}$ turns out to be independent of the particular $x^{*}$ for which it is defined.

We adapt the requirements on $\succsim$ encountered in section 3.2 to the new domain $H$. In the interests of space, we list them here, but state them formally in appendix $\mathrm{A}^{16}$ We require that $\succsim$ be complete, transitive, and continuous (Axiom X2), and satisfy Independence (Axiom X3) and Stationarity (Axiom X6). The remaining requirements on $\succsim$ are most easily stated as requirements on $\succsim s$. We require that $\succsim_{s}$ be non-trivial (Axiom X1), and satisfy Monotonicity (Axiom X4), Separability (Axiom X5), and Singleton Indifference to Timing (Axiom X7).

If all uncertainty is captured by the objective state, then $\succsim_{s}$ must be strategically rational. This results in a recursive version of the state dependent representation in Anscombe and Aumann [1963] (see corollary 6 below). In the presence of unobservable shocks, strategic rationality will be violated, even contingent on the state. The central assumption of this section requires those unobservable shocks to be transient. That is, DM must be strategically rational with respect to continuation problems, contingent on the state.

Aхıом X8 (State Contingent CSR). $\{(k, f)\} \succsim_{s}\{(k, g)\}$ implies $\{(k, f),(k, g)\} \sim_{s}$ $\{(k, f)\}$.

The axiom considers the choice of a menu contingent on $s$. As in CSR, all menus compared here fix next period's consumption in state $s$ at $k$. The axiom says that, contingent on $s$, there is no preference for flexibility with respect to continuation problems. This requires all persistent shocks that are relevant for future consumption tastes to be commonly observed (ie, captured by the state of the world), while allowing additional unobserved transient taste shocks.

The last axiom assumes that the state space is large enough, such that the current objective state captures all past persistent shocks.

(16) Axioms in this section are labelled with an ' $X$ ' to indicate the presence of exogenous states in the environment. The numbering of the axioms corresponds to those in the PFC representation. 
Аххом X9 (History Independence). $\left\{\left(k, f_{s}^{x}\right)\right\} \succsim_{s^{\prime}}\left\{\left(k, f_{s}^{y}\right)\right\}$ implies $\left\{\left(k, f_{s}^{x}\right)\right\} \succsim_{s^{\prime \prime}}$ $\left\{\left(k, f_{s}^{y}\right)\right\}$ for all $s, s^{\prime}, s^{\prime \prime} \in S$.

In analogy to the definition of $x \succsim_{s} y$ as the DM's ranking of menu $x$ over $y$ contingent on the first state realization being $s$, one can interpret $\left\{\left(k, f_{s}^{x}\right)\right\} \succsim_{s^{\prime}}\left\{\left(k, f_{s}^{y}\right)\right\}$ as the DM's ranking of $x$ over $y$ contingent on the first and second state realizations being $s^{\prime}$ and $s$, respectively. The axiom says that this contingent preference is independent of the first state realization 17

\subsection{Preference for Flexibility with Exogenous States}

In this section, we provide a representation that features a subjective Markov process over objective states, as well as state contingent beliefs over consumption utilities.

Definition 4. Let $U$ be defined as above, and for each $s \in S$, let $\mu_{s}$ be a nice probability measure on (the Borel sigma-algebra of) $U$. Let $\Pi$ represent the transition probabilities for a fully connected Markov process on $S,{ }^{18}$ and let $\pi_{0}$ be the unique invariant measure of $\Pi$. Also, let $\delta \in(0,1)$. A preference $\succsim$ has a representation of Preference for Flexibility with Exogenous States $(\mathrm{PFX}),\left(\left(\mu_{s}\right)_{s \in S}, \Pi, \delta\right)$, if $V\left(\cdot, \pi_{0}\right):=\sum_{s} V(\cdot, s) \pi_{0}(s)$ represents $\succsim$, where $V$ is defined recursively as

$$
V(f, s)=\sum_{s \in S} \Pi\left(s, s^{\prime}\right)\left[\int_{u} \max _{p \in f(s)}\left[u\left(p_{k}\right)+\delta V\left(p_{h}, s^{\prime}\right)\right] \mathrm{d} \mu_{s^{\prime}}(u)\right]
$$

In the representation above, $V$ is linear on $H$, and $V\left(p_{h}, \cdot\right)$ denotes the linear extension (by continuity) of $V$ from $H$ to $\mathscr{P}(H)$, that is, $V\left(p_{h}, \cdot\right)=\int V(g, \cdot) \mathrm{d} p_{h}(g)$. Notice that $V\left(f, \pi_{0}\right)$ takes the especially simple form

$$
V\left(f, \pi_{0}\right)=\sum_{s} \pi_{0}(s) \int_{u} \max _{p \in f(s)}\left[u\left(p_{k}\right)+\delta V\left(p_{h}, s\right)\right] \mathrm{d} \mu_{s}(u)
$$

which follows from the fact that $\pi_{0}\left(s^{\prime}\right)=\sum_{s} \pi_{0}(s) \Pi\left(s, s^{\prime}\right)$ is the invariant distribution of $\Pi$.

Proposition 5. Each PFX representation $\left(\left(\mu_{s}\right)_{s \in S}, \Pi, \delta\right)$ induces a unique continuous function $V \in C(H \times S)$ that satisfies equation (4.1) above.

(17) In Theorem 2 below, objective states follow a subjective Markov process. Relaxing History Independence (Axiom X9) would permit more complicated dynamics of the objective state. In the more familiar context of objective uncertainty states are routinely assumed to follow a Markov process, and this is justified by assuming that the state space is sufficiently large.

(18) The Markov process on $S$ is fully connected if $\Pi\left(s, s^{\prime}\right)>0$ for all $s, s^{\prime} \in S$. A weaker requirement would be that every state is irreducible. The class of fully connected Markov processes is dense in the class of irreducible processes. The small gain in generality does not seem to warrant imposing a weaker, but unfalsifiable version of History Independence, that only requires the existence of a finite number of state realizations, contingent on which the previous history of states is irrelevant. 
The proof is in appendix $\mathrm{E}$

TheOrem 2. The binary relation $\succsim$ satisfies Axioms X1 $X 9$ if, and only if, it has a PFX representation, $\left(\left(\mu_{s}\right)_{s \in S}, \Pi, \delta\right)$. Moreover, the measures $\left(\mu_{s}\right)_{s \in S}$ are unique up to a common scaling, and $\Pi$ and $\delta$ are unique.

The proof is in appendix $\mathrm{E}$ and a sketch can be found in section 4.3. The PFC representation is a special case of the PFX representation when $|S|=1$. Thus, Theorem 1 follows immediately from Theorem 2. As mentioned above, another interesting special case is the situation where each $\succsim_{s}$ is fully strategically rational. In that case the model reduces to a recursive version of the state dependent Anscombe and Aumann [1963] model, where DM has a preference for flexibility only because he is unsure about which state $s \in S$ will obtain and because his consumption utility $u_{s} \in \mathcal{U}$ depends on the state.

Corollary 6. Suppose $\succsim$ has a PFX representation $\left(\left(\mu_{s}\right)_{s \in S}, \Pi, \delta\right)$. Then, each $\succsim_{s}$ is strategically rational if, and only if, $\succsim$ has a recursive state dependent AnscombeAumann representation. That is, there is a collection $\left(u_{s}\right)_{s \in S} \subset \mathcal{U}$ where $\mu_{s}\left(u_{s}\right)=1$ for all $s \in S$, such that $V\left(\cdot, \pi_{0}\right):=\sum_{s} \pi_{0}(s) V(\cdot, s)$ represents $\succsim$, where $V$ is defined recursively as

$$
V\left(f, s^{\prime}\right)=\sum_{s} \Pi\left(s^{\prime}, s\right) \max _{p \in f(s)}\left[u_{s}\left(p_{k}\right)+\delta V\left(p_{h}, s\right)\right]
$$

Moreover, the set $\left(u_{s}\right)_{s \in S}$ is unique up to a common scaling, and $\Pi$ and $\delta$ are unique.

Identification of subjective probabilities on the state space in the standard (static) Anscombe-Aumann model requires two assumptions. First, the ordinal ranking of lotteries should be independent of the state. This assumption is referred to as the State Independence Axiom. Second, the cardinal representation of these rankings (the utility) should be normalized to be state independent. In contrast, the recursive state dependent Anscombe-Aumann representation of corollary 6 features instantaneous as well as continuation utilities that are state dependent. Recursivity with a constant discount factor is the only normalization needed to imply that beliefs, generated by a subjective Markov process, and state dependent utilities are fully identified.

We end with the observation (without proof) that requiring full CSR would imply that the Markov process generated by $\Pi$ is iid.

Corollary 7. Suppose $\succsim$ has a PFX representation $\left(\left(\mu_{s}\right)_{s \in S}, \Pi, \delta\right)$. Then, $\succsim$ satisfies CSR — ie, $\{(k, f)\} \succsim\{(k, g)\}$ implies $\{(k, f)\} \sim\{(k, f),(k, g)\}$ - if, and only if, $\Pi$ represents an iid process wherein $\Pi(s, \cdot)=\Pi\left(s^{\prime}, \cdot\right)$ for all $s, s^{\prime} \in S$.

\subsection{Proof Sketch for the PFX Representation}

Independence (Axiom X3) implies that there exists a representation of $\succsim$ of the form $V(f):=\sum_{s} \pi_{0}(s) U_{s}(f(s))$, where $U_{s}: \mathscr{F} \rightarrow \mathbb{R}$ is linear, continuous, and monotone 
(with respect to set inclusion). It is easy to see that $U_{s}$ represents $\succsim_{s}$. Theorem 4 (appendix B.1) provides a finitely additive EU representation for $\succsim_{s}$. Proposition 21 in the appendix shows that because $\succsim_{s}$ also satisfies Separability (Axiom X5), $U_{s}$ must take the form

$$
U_{s}(x)=\int_{\mathfrak{U}_{K} \times[0,1] \times \mathfrak{U}_{H}} \max _{p \in x}\left[\lambda r\left(p_{k}\right)+(1-\lambda) v_{s}\left(p_{z}\right)\right] \mathrm{d} \mu_{s}^{\prime}(r, \lambda, v)
$$

where $\mathfrak{U}_{K}$ and $\mathfrak{U}_{H}$ are spaces of twice-normalized $\mathrm{vN}-\mathrm{M}$ functions over $K$ and $H$, respectively. We show in appendix B.2 that any preference relation over menus that is represented by integration against a normal charge (see footnote 23 for a definition) is strategically rational if, and only if, the carrier ${ }^{19}$ of the charge is a singleton. Hence, State Contingent CSR (Axiom X8) implies that we can drop $\mathfrak{U}_{H}$ from the description of the state space. Next, lemma 23 shows that since the carrier of the normal charge $\mu^{\prime}$ is compact, $\mu^{\prime}$ can be extended to the (Borel) sigma-algebra of $\mathfrak{U}_{K} \times[0,1]$ as a (countably additive) probability measure. The preference functional then takes the form

$$
U_{s}(x)=\int_{\mathfrak{U}_{K} \times[0,1]} \max _{p \in x}\left[\lambda r\left(p_{k}\right)+(1-\lambda) v_{s}\left(p_{z}\right)\right] \mathrm{d} \mu_{s}^{\prime}(r, \lambda)
$$

Now consider the restricted collection of pairs of consumption and continuation problems $B:=K \times\left\{g_{\circ}, g^{\circ}\right\}$, where $g_{\circ}=\arg \min _{g \in H} v_{s}(g)$ and $g^{\circ}=$ $\arg \max _{g \in H} v_{s}(g)$, and notice that $B \subset K \times H$. Let $\iota: \mathscr{F}(\mathscr{P}(B)) \rightarrow \mathscr{F}(\mathscr{P}(K \times H))$ be the natural inclusion map identifying closed subsets of $\mathscr{P}(B)$ with closed subsets of $\mathscr{P}(K \times H)$. Define the restricted preference functional on $\mathscr{F}(\mathscr{P}(B))$ as $W_{s}(x)=U_{s}(\iota x)$ for all $x \in \mathscr{F}(\mathscr{P}(B))$. Since $B$ is finite, we may invoke the additive EU representation theorem of DLR, which says that for $W_{s}$, and therefore for $U_{s}$, beliefs and utilities are jointly identified. That is, they are identified up to an arbitrary normalization, as discussed after Theorem 1 .

Because it can be shown that $v_{s}$ is not constant on $\left\{g_{\circ}, g^{\circ}\right\}$, and because there is no preference for flexibility with respect to continuation problems, choice on $\mathscr{F}(\mathscr{P}(B))$ allows us to be cognizant of the tradeoff between instantaneous consumption from $K$ and future consumption from $H$. This tradeoff determines $\mu_{s}^{\prime}$ up to scaling. To see this, use the transformation $\frac{\lambda}{1-\lambda} r \mapsto u \in \mathcal{U}$ (and transform the measure $\mu_{s}^{\prime}$ on $\mathfrak{U}_{K} \times[0,1]$ appropriately to become a measure $\mu_{s}$ on $\mathcal{U}$ ), so that the preference functional $U_{s}$ can be written as

$$
U_{s}(x)=\int_{u} \max _{p \in x}\left[u\left(p_{k}\right)+v_{s}\left(p_{z}\right)\right] \mathrm{d} \mu_{s}(u)
$$

Suppose that $U_{s}^{*}(x)=\int_{U} \max _{p \in x}\left[u\left(p_{k}\right)+v_{s}^{*}\left(p_{z}\right)\right] \mathrm{d} \mu_{s}^{*}(u)$ is another preference functional that represents $\succsim_{s}$. Since beliefs and utilities are jointly identified, there

(19) Intuitively, the carrier of a charge is the smallest closed set that contains all the weight of the charge. If the charge is also a measure, the carrier is referred to as the support of the measure. See definition 13 in the appendix. 
exist functions $(\zeta, \xi): \mathcal{U} \mapsto \mathbb{R}_{+} \times \mathbb{R}$, such that for each $u^{*}$ in the support of $\mu_{s}^{*}$, we have $u^{*}\left(p_{k}\right)+v_{s}^{*}\left(p_{z}\right)=\zeta(u)\left[u\left(p_{k}\right)+v\left(p_{z}\right)\right]+\xi(u)$ for some $u$ in the support of $\mu$.

Consider two lotteries $p$ and $q$ such that $p_{k}=q_{k}$ but $v\left(p_{z}\right) \neq v\left(q_{z}\right)$. Then,

$$
\begin{aligned}
& \left(u^{*}\left(p_{k}\right)+v_{s}^{*}\left(p_{z}\right)\right)-\left(u^{*}\left(q_{k}\right)+v_{s}^{*}\left(q_{z}\right)\right) \\
& \quad=v_{s}^{*}\left(p_{z}\right)-v_{s}^{*}\left(q_{z}\right)=\zeta(u)\left(v_{s}\left(p_{z}\right)-v_{s}\left(q_{z}\right)\right)
\end{aligned}
$$

which implies that $\zeta(u)$ is independent of $u$ and hence is constant. Disregarding the additive constants, $\xi(u)$, we can write $U_{s}^{*}(x)=\int_{u} \max _{p \in x}\left[\zeta u\left(p_{k}\right)+\right.$ $\left.\zeta v\left(p_{z}\right)\right] \mathrm{d} \mu_{s}^{*}(\zeta u)$. Joint identification of beliefs and utilities implies that $\mu_{s}^{*}(\zeta u)=$ $\mu_{s}(u)$. That is, $\mu_{s}$ is unique up to scaling.

We have thus far established that $\succsim$ has a representation of the form

$$
V\left(f, \pi_{0}\right)=\sum_{s} \pi_{0}(s)\left[\int_{u} \max _{p \in f(s)}\left[u\left(p_{k}\right)+v_{s}(h)\right] \mathrm{d} \mu_{s}(u)\right]
$$

where each $\mu_{s}$ is identified up to scaling. Let $\succsim_{s}^{*}$ be the preference relation on $H$ induced by $v_{s}$. In appendix E.1.3, we show that our assumptions on $\succsim$ imply that $\succsim_{s}^{*}$ is continuous and satisfies Independence (Axiom X3). By the Mixture Space Theorem, $v_{s}$ is unique up to scaling and can be written as $v_{s}(f):=\sum_{\tilde{s}} w_{s, \tilde{s}}(f(\tilde{s}))$. By History Independence (Axiom X9), $w_{s, \tilde{s}}$ and $w_{s^{\prime}, \tilde{s}}$ are identical up to scaling. By Aggregate Stationarity (Axiom X6) they must then agree up to scaling with $U_{\tilde{s}}$. Hence, there is a scaling $\pi_{s} \in \mathbb{R}_{++}^{S}$, such that $v_{s}(f)=\delta_{s} \sum_{\tilde{s}} \pi_{s}(\tilde{s}) U_{\tilde{s}}(f(\tilde{s}))$, where $\delta_{s}>0$ is a scaling factor that normalizes $\pi_{s}$ to be a probability measure.

Defining $V(f, s):=v_{s}(f) / \delta_{s}$ and noting that $U_{\tilde{s}}$ can be written as in (4.2), we find the following recursive representation of $\succsim$ where the discount factor may only depend on the state:

$$
V\left(f, \pi_{0}\right)=\sum_{s} \pi_{0}(s)\left[\int_{u} \max _{p \in f(s)}\left[u\left(p_{k}\right)+\delta_{s} V\left(p_{h}, s\right)\right] \mu_{s}(\mathrm{~d} u)\right]
$$

represents $\succsim$, where

$$
V\left(f, s^{\prime}\right)=\sum_{s} \pi_{s^{\prime}}(s)\left[\int_{u} \max _{p \in f(s)}\left[u\left(p_{k}\right)+\delta_{s} V\left(p_{h}, s\right)\right] \mathrm{d} \mu_{s}(u)\right]
$$

and $\Pi\left(s^{\prime}, s\right):=\pi_{s^{\prime}}(s)$ is a fully connected Markov process on $S$.

It remains to establish that there is a unique renormalization of the value function $V$ such that the discount factors $\delta_{s}$ are independent of $s$, and that the corresponding transformation of $\pi_{0}$ is the unique invariant distribution of the transformed Markov process.

In the value function $V$, each $\mu_{s}$ is identified up to scaling. Let $\xi \in \mathbb{R}_{++}^{S}$ parametrize an arbitrary rescaling of the measures $\mu_{s}$, where the re-scaled measure 
is denoted by $\hat{\mu}_{s}(D)=\mu_{s}(D / \xi(s))$ for all measurable $D \subset \mathcal{U}$. Joint identification implies that if we re-scale the measures $\mu_{s}$ to become $\hat{\mu}_{s}$, we must also re-scale continuation utilities as well as subjective beliefs over exogenous states as follows: divide $\delta_{s} V_{s}$ by $\xi(s)$, and transform the subjective beliefs over exogenous states as $\hat{\pi}_{s^{\prime}}(s):=\pi_{s^{\prime}}(s) \xi(s) /\left\langle\pi_{s^{\prime}}, \xi\right\rangle$, where dividing by $\left\langle\pi_{s^{\prime}}, \xi\right\rangle$ ensures that $\hat{\pi}_{s^{\prime}}$ is a probability measure. Then,

$$
\sum_{s} \hat{\pi}_{0}(s)\left[\int_{u_{K}} \max _{p \in x}\left[u(s)+\frac{\delta_{s}}{\xi(s)} V\left(p_{e}, \pi_{s}\right)\right] d \hat{\mu}_{s}(u)\right]=\frac{V\left(f, \pi_{0}\right)}{\left\langle\pi_{0}, \xi\right\rangle}
$$

also represents $\succsim$.

In order to write this representation in the form of (4.3), define $\hat{V}\left(\cdot, \hat{\pi}_{0}\right):=$ $V\left(\cdot, \pi_{0}\right) /\left\langle\pi_{0}, \xi\right\rangle, \hat{V}\left(\cdot, \hat{\pi}_{s}\right):=V\left(\cdot, \pi_{s}\right) /\left\langle\pi_{s}, \xi\right\rangle$ and $\hat{\delta}_{s}:=\delta_{s}\left\langle\pi_{s}, \xi\right\rangle / \xi(s)$ to arrive at

$$
\hat{V}\left(f, s^{\prime}\right)=\sum_{s} \hat{\pi}_{s^{\prime}}(s)\left[\int_{u_{K}} \max _{p \in f(s)}\left[u(p)+\hat{\delta}_{s} \hat{V}(f, s)\right] \mathrm{d} \hat{\mu}_{s}(u)\right]
$$

where $\hat{V}\left(f, \hat{\pi}_{0}\right)$ as defined in (4.3) represents $\succsim$.

Thus, to show that there exists a unique representation with a constant discount factor, it suffices to show that there exists a unique $\xi \in \mathbb{R}_{++}^{S}$ such that $\hat{\delta}_{s}=\delta_{s}\left\langle\pi_{s}, \xi\right\rangle / \xi(s)$ is positive and independent of $s$. Recall that row $s$ of the Markov transition matrix $\Pi$ is $\pi_{s}$. Define the diagonal matrix $\Delta$ as follows:

$$
\Delta:=\left[\begin{array}{cccc}
\delta_{1} & 0 & \ldots & 0 \\
0 & \delta_{2} & \ldots & 0 \\
\vdots & \vdots & \ddots & \vdots \\
0 & 0 & \ldots & \delta_{n}
\end{array}\right]
$$

where $S=\{1, \ldots, n\}$. In matrix notation, our problem amounts to finding a $\xi \gg \mathbf{0}$ and $\hat{\delta}>0$ such that $\hat{\delta} \xi=\xi \Delta \Pi$. But this amounts to showing that (i) $\xi$ is a (left) eigenvector of the matrix $\Delta \Pi$, and (ii) $\hat{\delta}$ is the corresponding eigenvalue.

A matrix is positive if each of its entries is strictly positive. The matrix $\Pi$ is positive, so the matrix $\Delta \Pi$ is also positive. Thus, by the Perron theorem (Theorem 5 below), such $\xi$ and $\hat{\delta}$ exist, $\xi$ is unique up to scaling, and $\hat{\delta}$ is unique. This establishes that the collection of measures in $\left(\mu_{s}\right)_{s \in S}$ are unique up to a common scaling.

We emphasize that the continuation utilities in (4.3) and (4.4) are state dependent, and therefore cannot play the role of a numeraire. Instead, the key to the identification is the recursive structure of the representation in (4.4), that relates all the functions $V(\cdot, s)$ by the Bellman equation. To gain some intuition, consider the example of two PFR representations of the same preferences, $V$ and $\hat{V}$ as above, where $\delta$ and $\hat{\delta}$ are constant and, without loss of generality, $1>\delta \geqslant \hat{\delta}$. We established above that $\hat{\delta} \xi(s)=\delta\left\langle\pi_{s}, \xi\right\rangle$ must hold for all $s \in S$. Suppose $\xi\left(s^{\prime}\right) \neq \xi\left(s^{\prime \prime}\right)$ for some $s^{\prime}, s^{\prime \prime} \in S$. Pick the $s$ that minimizes $\xi(s)$ and observe that $\xi(s)<\left\langle\pi_{s}, \xi\right\rangle \leqslant \frac{\delta}{\delta}\left\langle\pi_{s}, \xi\right\rangle$, 
a contradiction. Hence, $\xi\left(s^{\prime}\right)=\xi\left(s^{\prime \prime}\right)$ for all $s^{\prime}, s^{\prime \prime} \in S$, which just says that $\mu_{s}$ and $\hat{\mu}_{s}$ are identical up to scaling.

To better understand why History Independence (Axiom X9) is necessary, we now provide an example of a preference that satisfies all axioms except Axiom X9. and show that it does not have a PFX representation. Suppose $\succsim$ can be represented by the value function $V$ in (4.3) above where $S=\{1,2\}$, and $\Pi=\left[\begin{array}{ll}1 & 0 \\ 0 & 1\end{array}\right]$, $\pi_{0}=\left(\frac{1}{2}, \frac{1}{2}\right)$, and $\delta_{1} \neq \delta_{2}$. The interpretation is that today DM is uncertain about tomorrow's exogenous state, but once he learns the state, he does not expect it to ever change again. In that case, there is no representation with a constant discount factor, because $\frac{\delta_{s}}{\xi(s)}\left\langle\pi_{s}, \xi\right\rangle=\delta_{s}$ for all positive $\xi\left(\right.$ where $\left.\pi_{s}=\Pi(s, \cdot)\right)$.

\section{Behavioral Comparison}

Preference for flexibility is the preference for non-degenerate menus over singletons. Intuitively, one decision maker has more preference for flexibility than another if he has a stronger preference for menus over singletons. To make matters precise, let us consider the restricted domain $L \subset Z$ of Infinite Horizon Consumption Streams (IHCSs). This domain consists of lotteries that deliver consumption for the present period and an IHCS for the next period. It is easy to show that $L$ is a closed and convex subset of $Z$. In a manner analogous to the characterization of risk aversion where lotteries are compared to certain amounts of money, characterizing preference for flexibility requires a comparison between IHCPs and IHCSs. This comparison is meaningful only if the preference restricted to $L$ is non-trivial.

Ахгом 9 (Consumption Non-triviality). There exist $\ell, \ell^{\prime} \in L$ such that $\ell \succ \ell^{\prime}$.

If $\succsim$ has a PFC representation, the restriction of $\succsim$ to $L$ is generated by DM's expected vN-M function $\mu u$. (Recall our notational convention whereby $\mu u:=\int_{u} u \mathrm{~d} \mu(u)$.) It then follows that if $\succsim$ has a PFC representation, it satisfies Consumption Non-triviality (Axiom 9 ) if, and only if, $\mu u \neq \mathbf{0}$ (where $\mathbf{0} \in \mathcal{U}$ ) 20

Definition 8. ${ }^{*}$ has greater preference for flexibility than $\succsim$ if

$$
x \succsim \ell \text { implies } x \succsim^{*} \ell
$$

for all $\ell \in L$ and $x \in Z 21$

(20) The "if" part of the claim is easy to see. To see the "only if" part, let $\mu$ have $\mu u=\mathbf{0}$, and consider $W \in C(Z)$ such that $W(\ell)=0$ for all $\ell \in L$ and $W(x) \geqslant 0$ for all $x \in Z$. Then, with $\Phi: C(Z) \rightarrow C(Z)$ given by $\Phi W(x):=\int_{u} \max _{p \in x}\left[u\left(p_{k}\right)+\delta W\left(p_{z}\right)\right] \mathrm{d} \mu(u)$, we have $\Phi W(x) \geqslant 0$ for all $x \in Z$, with equality on $L$. Therefore, the unique fixed point of $\Phi$, namely the value function $V$ representing $\succsim$, must also have this property.

(21) The definition is identical to the definition of more averse to commitment in HHT. 
The comparison in the definition implies that $\succsim$ and $\succsim^{*}$ have the same ranking of IHCSs, ie, $\ell \succsim \ell^{\prime}$ if, and only if, $\ell \succsim^{*} \ell^{\prime}$. (This is lemma 34 in the appendix and also assumes Independence.) Since preference for flexibility is the behavioral manifestation of uncertainty about consumption utilities, it is ideally characterized in terms of beliefs, which requires the following definition.

Definition 9 (Dilation). Let $Q(u, D)$ be a Markov kernel from $u$ to itself. Then $Q(u, D)$ is a dilation if it is expectation preserving, ie, if $\int_{u} u^{\prime} Q\left(u, \mathrm{~d} u^{\prime}\right)=u$ for each $u \in \mathcal{U}$. If $\mu$ and $\mu^{*}$ are probability measures on $\mathcal{U}$, then $\mu^{*}$ is a dilation of $\mu$ if there exists a dilation $Q$ such that $\mu^{*}=Q \mu$, ie, $\mu^{*}\left(\mathrm{~d} u^{\prime}\right):=\int Q\left(u, \mathrm{~d} u^{\prime}\right) \mu(\mathrm{d} u)$.

If $\mu^{*}$ is a dilation of $\mu$, then $\mu^{*} u=\mu u$, because a dilation preserves expectations.

The PFC representation $(\mu, \delta)$ only identifies the measure $\mu$ up to scaling. In order to facilitate a comparison of measures, we shall say that a PFC representation $(\mu, \delta)$ is canonical if $\|\mu u\|_{2}=1$. Obviously, $\succsim$ admits a canonical PFC representation if, and only if, $\mu u \neq \mathbf{0}$ which, in turn, holds if, and only if, $\succsim$ satisfies Axiom 9.

TheOREM 3. Let $\succsim$ and ${ }^{*}$ have canonical PFC representations $(\mu, \delta)$ and $\left(\mu^{*}, \delta^{*}\right)$, respectively. Then, the following are equivalent:

(a) $*^{*}$ has greater preference for flexibility than $\succsim$.

(b) $\delta=\delta^{*}$ and $\mu^{*}$ is a dilation of $\mu$.

The proof is in appendix F. Intuitively, DM* with preference ${ }^{*}$ has greater preference for flexibility than DM with preference $\succsim$ precisely because he expects more uncertainty to resolve before making a choice, which increases the option value of waiting to make a choice from the menu. Some asset pricing implications of Theorem 3 are explored in Krishna and Sadowski [2012a].

It is worthwhile to consider the case where $\succsim$ has a PFC representation but does not satisfy Consumption Non-triviality (Axiom 9). In that case DM is indifferent between all IHCSs, but nevertheless has a preference for flexibility, presumably because he expects to learn about his consumption utility in the future. It follows from Monotonicity (Axiom 4) that every menu $x$ is preferred to every IHCS $\ell \in L$. Obviously, our definition of greater preference for flexibility has no bite in that case.

As mentioned above, subjective uncertainty in the dynamic model of HHT only concerns the discount factor. Thus, their model is a special case of the PFC representation, where the support of the measure $\mu$ can be written as $\{\lambda u: \lambda>0\}$ for some $u \in \mathcal{u}$. Consequently, HHT's characterization of greater preference for commitment (their Theorem 4.2) is a special case of our Theorem 3 .

Finally, we emphasize that the behavioral comparison in terms of beliefs provided by Theorem 3 is possible only because beliefs are identified up to a scaling 
in our dynamic setting. In contrast, the notion of 'greater preference for flexibility' proposed in DLR for the static context cannot rely on beliefs because those are not identified in their model. Hence, instead of characterizing whether one DM has a stronger preference for flexibility than another, DLR characterize whether one DM has any preference for flexibility whenever the other does. Neither ranking is complete. While ours can only compare preferences that agree on the ranking of singletons, the ranking in DLR can only compare preferences with representations for which the support of the measure is ordered by set inclusion.

In the context of the PFX representation we can also compare DM's strength of preference for flexibility across states. We define a state contingent consumption stream as an act that gives, in each state, a lottery over the pair consisting of instantaneous consumption and a contingent continuation stream. A formal recursive definition is given in appendix C. This allows us to adapt Consumption Non-triviality (Axiom 9) to the domain $H$.

Proposition 10. Suppose $\succsim$ has a PFX representation and satisfies the appropriate version of Axiom 9. Then, for $s, s^{\prime} \in S$, the following are equivalent:

(a) $\succsim_{s}$ exhibits greater preference for flexibility than $\succsim_{s^{\prime}}$.

(b) $\mu_{s}$ is a dilation of $\mu_{s^{\prime}}$ and $\Pi(s, \cdot)=\Pi\left(s^{\prime}, \cdot\right)$.

The fact that $\succsim_{s}$ and $\succsim_{s^{\prime}}$ must be the same on the subdomain of consumption streams implies $\Pi(s, \cdot)=\Pi\left(s^{\prime}, \cdot\right)$. Hence, if all states are comparable in the sense of greater preference for flexibility, then objective states evolve according to a process that is iid.

Proposition 10 describes how DM trades off flexibility across states. For example, if condition (b) is satisfied for states $s$ and $s^{\prime}$, and if $\pi_{0}(s) \geqslant \pi_{0}\left(s^{\prime}\right)$, then DM prefers flexibility in state $s$ over flexibility in state $s^{\prime}$.

\section{Conclusion}

We provide foundations for uniquely identified recursive representations of preference for flexibility by relaxing the standard assumption of strategic rationality to accommodate unobservable taste shocks. If taste shocks are transient, strategic rationality with respect to continuation problems should be satisfied unconditionally (CSR). If there are transient and persistent taste shocks, but only transient shocks are unobservable, then strategic rationality with respect to continuation problems should hold contingent on the objective state of the world (State Contingent CSR).

What if persistent taste shocks are also unobservable? Then CSR should be satisfied only contingent on some history of past tastes and, in the simplest case, contingent on the current consumption taste. While this taste is by assumption 
unobservable, CSR would also have to hold contingent on current consumption choice from a large enough menu. In a supplement, Krishna and Sadowski [2012b], we pose an axiom called Choice Contingent CSR that captures this idea and derive a representation that can accommodate unobserved persistent taste shocks by allowing consumption utilities themselves to follow a particular, and uniquely identified Markov process. The proof builds on the proof for the PFX representation, where the collection of possible consumption rankings plays the role of the state space.

While we suggest the three versions of CSR above-namely CSR along with State Contingent and Choice Contingent CSR - as a natural starting point when trying to understand how strategic rationality is violated, these could obviously be relaxed further. For example, CSR might only be satisfied contingent on finite histories of consumption choices and states. Modelling the corresponding evolution of beliefs as a Markov process would require a larger subjective state space, as well as an assumption that ensures that preference for flexibility is sufficiently persistent. Beyond that, our proofs of identification only require that the implied Markov process over preferences have a unique stationary distribution. Given the persistence of preference for flexibility, such uniqueness is ensured by Stationarity (via an application of the Perron Theorem).

In that sense, we view our work as illustrative of how to achieve a fully identified recursive representation of choice when the assumption of strategic rationality is further weakened. 


\section{Appendices}

\section{A. Axioms from Section 4}

Aхıом X1 (Statewise Non-triviality). Every state $s$ is non-null, in the sense that there exist $x, y \in \mathscr{F}$ such that $x \succ_{s} y$.

Ахгом X2 (Continuous Order). $\succsim$ satisfies the following:

(a) $\succsim$ is complete and transitive.

(b) $\succsim$ is continuous, in the sense that the sets $\{f: f \succsim g\}$ and $\{f: g \succsim f\}$ are closed.

Axıом X3 (Independence). $f \succ g$ implies $\lambda f+(1-\lambda) h \succ \lambda g+(1-\lambda) h$ for all $\lambda \in(0,1)$.

Ахıом X4 (Monotonicity). $x \cup y \succsim_{s} x$ for all $x, y \in \mathscr{F}$.

Ахıом X5 (Separability). If, for $p, q \in \mathscr{P}(K \times H)$, the marginal distributions satisfy $p_{k}=q_{k}$ and $p_{h}=q_{h}$, then $\{p, q\} \sim_{s}\{p\}$.

Recall that $(k, f)$ denotes the degenerate lottery that gives $(k, f) \in K \times H$ with probability one in every state.

Ахıом Х6 (Aggregate Stationarity). $f \succsim g$ if, and only if, $\{(k, f)\} \succsim\{(k, g)\}$.

Aхıом X7 (Singleton Indifference to Timing). $\{\lambda(k, f)+(1-\lambda)(k, g)\} \sim_{s}\{(k, \lambda f+(1-$ $\lambda) g)\}$ for all $\lambda \in[0,1]$.

\section{B. Abstract Representations}

We shall first construct a general representation in the spirit of DLRS where the prize space is infinite. We then study the effect of assuming Strategic Rationality. We begin by defining and collecting some facts about support functions.

Let $Y$ be a compact metric space. Let $\mathscr{P}(Y)$ be the space of probability measures on $Y$, endowed with the topology of weak convergence, which makes $\mathscr{P}(Y)$ compact and metrizable, and let $C(Y)$ denote the Banach space of uniformly continuous functions on $Y$. In what follows, for $f \in C(Y)$ and $p \in \mathscr{P}(Y)$, we will frequently denote $\int f \mathrm{~d} p$ by $f(p)$. Fix $p^{*} \in \mathscr{P}(Y)$, and let $X:=\left\{f \in C(Y): f\left(p^{*}\right)=0\right\}$.

For any weak* closed, convex subset $G$ of $\mathscr{P}(Y)$, let $\bar{h}_{G}: X \rightarrow \mathbb{R}$ be its extended support function, given by $\bar{h}_{G}(f):=\sup _{p \in G} f(p)$. The extended support function is sublinear, ie, subadditive and positively homogeneous, and Mackey continuous [Theorem 5.102, Aliprantis and Border, 1999], and hence is also norm continuous, since the Mackey and norm topology coincide on normed spaces [Corollary 6.27, Aliprantis and Border, 1999].

Let $\mathfrak{U}_{Y}:=\left\{f \in X:\|f\|_{\infty}=1\right\}$, and notice that an extended support function is completely defined by the values it takes on $\mathfrak{U}_{Y}$. Therefore, for any sublinear and norm continuous function $\bar{h}: X \rightarrow \mathbb{R}$, we shall consider its restriction to $\mathfrak{U}_{Y}$, denoted by $h$. We call a function $h: \mathfrak{U}_{Y} \rightarrow \mathbb{R}$ a support function if its unique extension to $X$ by positive 
homogeneity is sublinear and norm continuous, ie, if it is an extended support function in the sense described above.

Support functions have the following duality: For any weak* compact, convex subset $G$ of aff $(\mathscr{P}(Y)), G_{h_{G}}=G$ where $G_{h_{G}}:=\left\{p \in\right.$ aff $(\mathscr{P}(Y)): f(p) \leqslant h_{G}(f)$ for all $\left.f \in \mathfrak{U}_{Y}\right\}$. Support functions also have the following useful properties ${ }^{22}$ for weak ${ }^{*}$ compact, convex subsets $G, H$ of $\mathscr{P}(Y)$ : (i) $G \subset H$ if and only if $h_{G} \leqslant h_{H}$, (ii) $h_{t G+(1-t) H}=t h_{G}+(1-t) h_{H}$ for all $t \in(0,1)$, (iii) $h_{G \cap H}=h_{G} \wedge h_{H}$, and (iv) and $h_{\operatorname{conv}(G \cup H)}=h_{G} \vee h_{H}$. (Since $G$ and $H$ are convex, it follows from Lemma 5.14 of Aliprantis and Border [1999] that $\operatorname{conv}(G \cup H)$ is also compact.) Notice also that $h_{\left\{p^{*}\right\}}=\mathbf{0}$.

\section{B.1. Constructing a General Representation}

Let $Y$ be a compact metric space of prizes, so $\mathscr{P}(Y)$ is a compact metric space. Let $\mathscr{F}(\mathscr{P}(Y))$ denote the space of compact subsets of $\mathscr{P}(Y)$, and $\mathscr{K}(\mathscr{P}(Y))$ the space of closed, convex subsets of $\mathscr{P}(Y)$, both endowed with the Hausdorff metric. Then, $\mathscr{K}(\mathscr{P}(Y))$ is a closed subspace of $\mathscr{F}(\mathscr{P}(Y))$. We consider a preference $\succsim$ over $\mathscr{F}(\mathscr{P}(Y))$. For notational ease, we shall write $\mathscr{F}$ and $\mathscr{K}$ for $\mathscr{F}(\mathscr{P}(Y))$ and $\mathscr{K}(\mathscr{P}(Y))$ respectively. Typical elements of $\mathscr{F}$ will be denoted by $G, G^{\prime}$ etc. As always, $\alpha G+(1-\alpha) G^{\prime} \in \mathscr{F}$ is the Minkowski sum.

The space of all vN-M utility functions is simply $C(Y)$, the Banach space of uniformly continuous functions on $Y$. In general, in a state dependent additive EU representation, the vN-M utility functions need only be identified up to positive affine transformation. Fix $p^{*} \in \mathscr{P}(Y)$ as above and, analogous to DLRS, let the subjective state space be given by $\mathfrak{U}_{Y}:=\left\{f \in C(Y): f\left(p^{*}\right)=0\right.$ and $\left.\|f\|_{\infty}=1\right\}$, the space of all $\mathrm{vN}-\mathrm{M}$ utility functions that (i) take the value 0 at $p^{*}$, (ii) are nontrivial on $\mathscr{P}(Y)$, and (iii) lie on the boundary of the unit ball of $C(Y)$.

We refer to the state space $\mathfrak{U}_{Y}$ as the canonical state space. Let $\mathscr{A}_{\mathfrak{U}_{Y}}$ be the Borel algebra of sets in $\mathfrak{U}_{Y}$, and $\mu$ a normal ${ }^{23}$ charge on $\left(\mathfrak{U}_{Y}, \mathcal{A}_{\mathfrak{U}_{Y}}\right)$. A pair $\left(\mathfrak{U}_{Y}, \mu\right)$ is a finitely additive EU representation of $\succsim$ if $V(x)=\int_{\mathfrak{U}_{Y}} \max _{p \in x} f(p) \mathrm{d} \mu(f)$ represents $\succsim$.

Theorem 4. A preference, ¿, satisfies Non-triviality, Continuous Order, Monotonicity, and Independence ${ }^{24}$ if, and only if, it admits a finitely additive EU representation.

Note the key differences between Theorem 4 and the additive EU representation theorem of DLR. They establish that given the subjective state space, the measure is unique and countably additive, while the theorem above establishes neither. There are two reasons for these differences. The first is that our subjective state space is not compact, therefore, the Riesz Representation Theorem only guarantees that the measure is finitely additive. (Notice that this would remain the case even if one were able to establish uniqueness of the representation.) The second difference is that we are unable to show that the span of the space of all support functions is dense in the space of all continuous bounded functions

(22) See, for instance, p 226 of Aliprantis and Border [1999].

(23) A charge is outer regular if every set in $\mathcal{A}_{\mathfrak{U}_{Y}}$ can be approximated from without by open sets; inner regular if it can be approximated from within by closed sets; and normal if it is both outer and inner regular - see also definition 10.2 in Aliprantis and Border [1999].

(24) These are the obvious extensions of Axioms $1,2,3$, and 4 to this general domain. 
on the subjective state space, a result that holds when the subjective state space is finite dimensional (and hence compact). Before the formal details are presented, we provide some intuition for the proof.

The proof of the representation naturally extends ideas in DLRS to the infinite dimensional setting. The first step is to show that each menu can be identified (isometrically) with its support function, and that support functions live in the space of twice normalized, non-trivial vN-M functions on the prize space $Y$. (This is exactly as in DLRS.) This allows us to define the subjective state space as a space of all twice normalized, continuous, non-constant, non-trivial functions on $Y$. Instead of looking at the space of menus, we can look at the space of support functions, a subset of all continuous bounded functions on the subjective state space.

The second step of the proof shows that any linear functional on the space of menus induces a continuous linear functional on the corresponding space of support functions. Moreover, since the preference $\succsim$ is monotone, this linear functional is Lipschitz, and can therefore be extended to the space of all continuous bounded functions on the subjective state space. (This step uses the Hahn-Banach Theorem.) The final step uses the Riesz Representation Theorem to show that any linear functional on the space of all continuous bounded functions can be written as an integral with respect to a finitely additive measure.

Proof of Theorem 4. By the continuity of $\succsim$, and since $\succsim$ satisfies Independence (Axiom 3 as applied to our domain), an adaptation of Lemmas 1 and 2 from DLR implies that $G \sim \overline{\operatorname{conv}}(G)$ for each $G \in \mathscr{F}$. (In particular, we have $\overline{\operatorname{conv}}\left(t G+(1-t) G^{\prime}\right)=t \overline{\operatorname{conv}}(G)+$ $(1-t) \overline{\operatorname{conv}}\left(G^{\prime}\right)$.) Following DLR, it suffices to restrict attention to $\mathscr{K}$, the space of all weak* compact, convex subsets of $\mathscr{P}(Y)$. Here, $\mathscr{P}(Y)$ is endowed with a metric inducing the weak* topology, and $\mathscr{K}$ is endowed with the Hausdorff metric. Let $K_{0}:=\left\{h \in C_{b}\left(\mathfrak{U}_{Y}\right): h=\right.$ $h_{G}$ for some $\left.G \in \mathscr{K}\right\}$ denote the affine embedding of $\mathscr{K}$ in $C_{b}\left(\mathfrak{U}_{Y}\right)$, where $C_{b}\left(\mathfrak{U}_{Y}\right)$ is the space of bounded and continuous functions on $\mathfrak{U}_{Y}$, endowed with the supremum norm, and recall that by construction, $\mathbf{0} \in K_{0}$.

Define the induced preference $\succsim^{*}$ on $K_{0}$, so that $G \succsim G^{\prime}$ if, and only if, $h_{G} \succsim^{*} h_{G^{\prime}}$. It is easily seen that $\succsim^{*}$ is complete and transitive, and satisfies continuity, Independence, and Monotonicity ${ }^{25}$ since $\succsim$ has these properties. By the Mixture Space Theorem (see, for instance, Fishburn [1970] or Kreps [1988]), there exists a function $\varphi: K_{0} \rightarrow \mathbb{R}$ that represents $\succsim^{*}$, is linear in the sense that $\left.\varphi\left(\alpha h^{1}+(1-\alpha) h^{2}\right)\right)=\alpha \varphi\left(h^{1}\right)+(1-\alpha) \varphi\left(h^{2}\right)$, and is positive so that $h^{1} \geqslant h^{2}$ implies $\varphi\left(h^{1}\right) \geqslant \varphi\left(h^{2}\right)$.

Let $K_{1}:=\bigcup_{r \geqslant 0} r K_{0}$ be the cone generated by $K_{0}$. Extend $\varphi$ to $K_{1}$ by positive homogeneity, and let $\Phi_{0}$ denote this extension. Notice that $\Phi_{0}$ is linear and positive.

Let us now consider $K_{1}-K_{1}$, and notice first that any $f \in K_{1}-K_{1}$ can be written as $r_{1}\left(h_{1}-h_{1}^{\prime}\right)$. Then, for any $f, g \in K_{1}$, we have $f-g=\left[r_{1}\left(h_{1}-h_{1}^{\prime}\right)\right]-\left[r_{2}\left(h_{2}-h_{2}^{\prime}\right)\right]=\left(r_{1} h_{1}+\right.$ $\left.r_{2} h_{2}\right)-\left(r_{1} h_{1}^{\prime}+r_{2} h_{2}^{\prime}\right)$. It follows that $f-g \in K_{1}-K_{1}$ since $\left(r_{1} h_{1}+r_{2} h_{2}\right),\left(r_{1} h_{1}^{\prime}+r_{2} h_{2}^{\prime}\right) \in K_{1}$. In particular, there exist $h_{G_{1}}, h_{G_{2}} \in K_{0}$ and $r \geqslant 0$ such that $f-g=r\left[h_{G_{1}}-h_{G_{2}}\right]$, from which it follows that $K_{1}-K_{1}$ is a vector subspace of $C_{b}\left(\mathfrak{U}_{Y}\right)$.

(25) Recall that $G \supset G^{\prime}$ if, and only if, $h_{G} \geqslant h_{G^{\prime}}$. Monotonicity of $\succsim$ requires that if $G \supset G^{\prime}$, then $G \succsim G^{\prime}$, which implies that we must have $h_{G} \succsim^{*} h_{G^{\prime}}$. Thus, $\succsim^{*}$ satisfies Monotonicity in the sense that for $h^{1}, h^{2} \in K_{0}, h^{1} \geqslant h^{2}$ implies $h^{1} \succsim^{*} h^{2}$. 
The linear function $\Phi_{0}$ can be extended to $K_{1}-K_{1}$ by linearity. As in DLRS, we claim that $\Phi_{0}$ on $K_{1}-K_{1}$ is Lipschitz. To see this, consider $f-g \in K_{1}-K_{1}$ such that $f-g \geqslant \mathbf{0}$. Then, $\Phi_{0}(f-g)=r \Phi_{0}\left(h_{G_{1}}-h_{G_{2}}\right)=r \Phi_{0}\left(h_{G_{1}}\right)-r \Phi_{0}\left(h_{G_{2}}\right)$. But by the positivity of $\varphi$, it follows that $\varphi\left(h_{G_{1}}\right) \geqslant \varphi\left(h_{G_{2}}\right)$, since $h_{G_{1}}-h_{G_{2}} \geqslant \mathbf{0}$ (ie, $\left.G_{1} \supset G_{2}\right)$. Therefore, it must be the case that $\Phi_{0}(f) \geqslant \Phi_{0}(g)$. Finally, notice that for any $f \in K_{1}-K_{1}$, we have $f \leqslant\|f\|_{\infty} 1$ (indeed, $|f| \leqslant\|f\|_{\infty} \mathbf{1}$, where $\mathbf{1}$ is the constant function equal to 1 ), so that $\Phi_{0}(f) \leqslant\|f\|_{\infty} \Phi_{0}(\mathbf{1})$, ie, $\Phi_{0}$ is Lipschitz with constant $\Phi_{0}(\mathbf{1})$.

Since $\Phi_{0}$ is Lipschitz on a vector subspace $K_{1}-K_{1}$, the Hahn-Banach Theorem allows us to extend it to $C_{b}\left(\mathfrak{U}_{Y}\right)$, with the extension being denoted by $\Phi$. The Riesz Representation Theorem [Theorem 13.9, Aliprantis and Border, 1999] allows us to represent the linear functional $\Phi$ on $C_{b}\left(\mathfrak{U}_{Y}\right)$ as an integral with respect to a normal charge $\mu$ on $\mathscr{A}_{\mathfrak{U}_{Y}}$, the algebra generated by the open sets of $\mathfrak{U}_{Y}$, as desired.

\section{B.2. Strategic Rationality}

Let $\succsim$ be a preference on $\mathscr{F}$.

Definition 11. A preference, $\succsim$, is strategically rational if $G \succsim G^{\prime}$ implies $G \sim G \cup G^{\prime}$.

In this section, we show that if $\succsim$ has a finitely additive EU representation $\left(\mathfrak{U}_{Y}, \mu\right)$, it is strategically rational if, and only if, $\mu$ is carried by a singleton. We begin with a lemma.

Lemma 12. For any $\mathfrak{U}_{0} \subset \mathfrak{U}_{Y}$, the following are equivalent:

(a) $\left|\mathfrak{U}_{0}\right|=1$.

(b) For all $p, q \in \mathscr{P}(Y)$ and for all $f_{1}, f_{2} \in \mathfrak{U}_{0}, f_{1}(p) \geqslant f_{1}(q)$ if and only if $f_{2}(p) \geqslant f_{2}(q)$.

Proof. It is clear that (a) implies (b). To see that (b) implies (a), notice that by the definition of $\mathfrak{U}_{Y}$, there exists $p^{*} \in \mathscr{P}(Y)$ such that $f\left(p^{*}\right)=0$ for all $f \in \mathfrak{U}_{Y}$. Moreover, $\|f\|_{\infty}=1$ for all $f \in \mathfrak{U}_{Y}$. Therefore, no $f_{1} \in \mathfrak{U}_{Y}$ is a positive affine transformation of some other $f_{2}$ in $\mathfrak{U}_{Y}$.

Now suppose $\left|\mathfrak{U}_{0}\right|>1$, and let $f_{1}, f_{2} \in \mathfrak{U}_{Y}$ be distinct. But by the definition of $\mathfrak{U}_{Y}, f_{1}$ and $f_{2}$ represent the same expected utility preference $\succsim^{*}$ on $\mathscr{P}(Y)$. Therefore, by the expected utility theorem and since $f_{1}\left(p^{*}\right)=f_{2}\left(p^{*}\right)=0, f_{1}=\alpha f_{2}$ for some $\alpha>0$, which contradicts the definition of $\mathfrak{U}_{Y}$, wherein $\|f\|_{\infty}=1$ for all $f \in \mathfrak{U}_{Y}$. This proves our claim.

Definition 13. The carrier of the charge $\mu$ is the set $\mathfrak{U}_{\mu}:=\bigcap\left\{N: N\right.$ is closed, $\left.\mu\left(N^{c}\right)=0\right\}$.

The carrier of the charge always exists, and is clearly well defined. If the charge is also a measure, then the carrier is referred to as the support of the measure. Moreover, given the definition of $\mu$, we have $\mathfrak{U}_{\mu} \subset \mathfrak{U}_{Y}$. For any $p, q \in \mathscr{P}(Y)$, define $\mathfrak{U}_{p, q}:=\left\{f \in \mathfrak{U}_{Y}: f(p)>\right.$ $f(q)\}$ and $\mathfrak{U}_{p, q}^{\circ}:=\left\{f \in \mathfrak{U}_{Y}: f(p)=f(q)\right\}$. Notice that $\mathfrak{U}_{p, q}$ is always open and $\mathfrak{U}_{p, q}^{\circ}$ is always closed, since $p$ is a continuous (linear) functional on $\mathfrak{U}_{Y}$, which is a closed set.

Lemma 14. If $\succsim$ on $\mathscr{F}$ is strategically rational, then $\min \left\{\mu\left(\mathcal{U}_{p, q}\right), \mu\left(\mathfrak{U}_{q, p}\right)\right\}=0$ for all $p, q \in \mathscr{P}(Y)$. 
Proof. Suppose to the contrary there exist $p, q$ with $\min \left\{\mu\left(\mathfrak{U}_{p, q}\right), \mu\left(\mathfrak{U}_{q, p}\right)\right\}=\mu\left(\mathfrak{U}_{p, q}\right)>0$. It is clear that $\{p, q\} \nsim\{p\},\{q\}$, which violates Strategic Rationality.

Lemma 15. If for all $p, q \in \mathscr{P}(Y), \min \left\{\mu\left(\mathfrak{U}_{p, q}\right), \mu\left(\mathfrak{U}_{q, p}\right)\right\}=0$, then $\left|\mathfrak{U}_{\mu}\right|=1$.

Proof. If for all $p, q \in \mathscr{P}(Y), \min \left\{\mu\left(\mathfrak{U}_{p, q}\right), \mu\left(\mathfrak{U}_{q, p}\right)\right\}=0$, then either (i) $\mathfrak{U}_{\mu} \subset \mathfrak{U}_{p, q} \cup \mathfrak{U}_{p, q}^{\circ}$, or (ii) $\mathfrak{U}_{\mu} \subset \mathfrak{U}_{q, p} \cup \mathfrak{U}_{p, q}^{\circ}$, but not both (by the definition of $\mathfrak{U}_{\mu}$ and since $\mathfrak{U}_{p, q}$ and $\mathfrak{U}_{q, p}$ are open).

In other words, for all $p, q \in \mathscr{P}(Y)$, and for all $f_{1}, f_{2} \in \mathfrak{U}_{Y}, f_{1}(p) \geqslant f_{1}(q)$ if, and only if, $f_{2}(p) \geqslant f_{2}(q)$. Lemma 12 now implies that $\left|\mathfrak{U}_{\mu}\right|=1$, as required.

We may now put all this together, as follows.

Proposition 16. Let $\succsim$ be a preference on $\mathscr{F}(\mathscr{P}(Y))$ that has a finitely additive representation $\left(\mathfrak{U}_{Y}, \mu\right)$. Then, the following are equivalent:

(a) $\succsim$ is strategically rational.

(b) The carrier of the charge $\mu$ is a singleton, ie, $\left|\mathfrak{U}_{\mu}\right|=1$.

Proof. It is easy to see that (b) implies (a). We shall now establish that (a) implies (b). By Lemma 14, strategic rationality implies $\min \left\{\mu\left(\mathfrak{U}_{p, q}\right), \mu\left(\mathfrak{U}_{q, p}\right)\right\}=0$ for all $p, q \in \mathscr{P}(Y)$. By Lemma 15 , we can then conclude that $\left|\mathfrak{U}_{\mu}\right|=1$, as desired.

\section{The Domain of SIHCPs}

We now sketch the construction of State Contingent Infinite Horizon Consumption Problems (SIHCPs) introduced in section 4. The IHCPs introduced in section 3 are a special case of this construction. The construction adapts ideas from GP and so we omit details. As in the text, $K$ is a finite set of consumption prizes in any period, and $S$ is a finite set of states.

Let $H_{1}:=\mathscr{H}(\mathscr{F}(\mathscr{P}(K)))$ denote the set of acts that give a closed subset of $\mathscr{P}(K)$ in each state $s \in S$. It is a compact metric space when endowed with the (product) Hausdorff metric. For each $t>1$, inductively define $H_{t}:=\mathscr{H}\left(\mathscr{F}\left(\mathscr{P}\left(K \times H_{t-1}\right)\right)\right)$, which is also a compact metric space. Thus, each $f_{t} \in H_{t}$ is an act that gives a closed set of probability measures over $K \times H_{t-1}$ in each state $s \in S$. This allows us to define the space $H^{*}:=$ $\mathrm{X}_{t=1}^{\infty} H_{t}$, which is also a compact metric space.

Thus, an element in $H^{*}$ is a collection $\left(f_{t}\right)$, where $f_{t} \in H_{t}$ for each $t \geqslant 1$. Each $f_{t}$ contains information about some of the $f_{\tau} \in H_{\tau}$ for all $\tau<t$ since each $p_{t} \in f_{t}(s)$ is a probability measure over $K \times H_{t-1}$, and each $p_{t-1} \in f_{t-1}(s)$ is a probability measure over $K \times H_{t-2}$, and so on. A sequence $\left(f_{t}\right)$ is consistent if, roughly speaking, the information in $f_{t}$ about $f_{t-2}$ is in accord with the information in $f_{t-1}$ about $f_{t-2}{ }^{26}$ The space of all consistent sequences, denoted by $H \subset H^{*}$, is the space of SIHCPs. A simple adaptation of Theorem A1 of GP shows that there is a homeomorphism between $H$ and $\mathscr{H}(\mathscr{F}(\mathscr{P}(K \times H)))$. In fact, it can be shown that this homeomorphism is an affine or linear homeomorphism, so

(26) See GP for a precise definition of consistency. It is easy to construct examples of sequences in $H^{*}$ that are not consistent. 
that we can define a linear preference on $H$ (ie, a preference that satisfies Independence) and study the naturally induced preference on $\mathscr{H}(\mathscr{F}(\mathscr{P}(K \times H)))$.

There are three special cases that are of importance to us. The first is the space of State Contingent Infinite Horizon Consumption Streams (SIHCSs) used in proposition 10, This is the subset $M \subset H$ that delivers a singleton menu in each state and in every period. It can be shown that $M \simeq \mathscr{H}(\mathscr{P}(K \times M))$. The second important case is where $S$ is a singleton, so that the agent always chooses between menus rather than acts. This defines a domain $Z \simeq \mathscr{F}(\mathscr{P}(K \times Z))$, which is the domain of IHCPs introduced in section 3 and used in the PFC representation, as well as the PFR representation in Krishna and Sadowski [2012b]. This is also the domain constructed in GP. The third case is the space of Infinite Horizon Consumption Streams (IHCSs) used in Theorem 3. This is the subset $L \subset Z$ that delivers a singleton menu in every period. It can be shown that $L \simeq \mathscr{F}(\mathscr{P}(K \times L))$.

\section{A Separable Representation}

In the process of obtaining our representations, we will frequently find it useful to obtain an intermediate representation on one state space, and then transform the representation so it is defined on another state space. The following lemma is an abstract version of this idea. In what follows, we consider the prize space $K \times Y$. If $Y$ is compact, $K \times Y$ is also compact. This allows us to define the canonical state space $\mathfrak{U}_{K \times Y}$ and with a typical state given by $\mathfrak{u} \in \mathfrak{U}_{K \times Y}$. Recall that $\mathfrak{U}_{K \times Y}=\left\{\mathfrak{u} \in C(K \times Y):\|\mathfrak{t}\|_{\infty}=1, \sum_{k \in K} \mathfrak{u}\left(k, p^{*}\right)=0\right\}$ for some lottery $p^{*} \in \mathscr{P}(Y)$. We say that a finitely additive EU representation $\left(\mathfrak{U}_{Y}, \mu\right)$ is jointly identified if, given the state space $\mathfrak{U}_{Y}$, the charge $\mu$ is unique ${ }^{27}$

Lemma 17 (Change of State Space). Let $\left(\mathfrak{U}_{K \times Y}, \mu\right)$ be a finitely additive EU representation of $\succsim$. A sufficient condition for $\left(\mathfrak{U}_{K \times Y}^{\prime}, \mu^{\prime}\right)$ to be another finitely additive EU representation of $\succsim$ is that there exist functions $\Psi: \mathfrak{U}_{K \times Y} \rightarrow \mathfrak{U}_{K \times Y}^{\prime}$ and $(\zeta, \xi): \mathfrak{U}_{K \times Y} \rightarrow \mathbb{R}_{++} \times \mathbb{R}$ such that $\Psi$ is a measurable bijection and $(\zeta, \xi)$ are integrable, and that satisfy:

(a) for all $\mathfrak{t}^{\prime}$ in the image of $\Psi, \mathfrak{t}^{\prime}(p)=\zeta(\mathfrak{u})(\Psi \mathfrak{u})(p)+\xi(\mathfrak{u})$ for all $p \in \mathscr{P}(K \times Y)$, and

(b) the bijection $\Psi$ is measure preserving, ie, for all measurable $D^{\prime} \subset \mathfrak{U}_{K \times Y}^{\prime}, \mu^{\prime}\left(D^{\prime}\right)=$ $\mu\left(\Psi^{-1} D^{\prime}\right)$, and for all measurable $D \subset \mathfrak{U}_{K \times Y}, \mu(D)=\mu^{\prime}(\Psi D)$.

If the finitely additive EU representation $\left(\mathfrak{U}_{K \times Y}, \mu\right)$ is jointly identified, then the condition is also necessary.

The proof of the sufficiency part of the lemma merely amounts to a change of variable, and is an instance of the nonuniqueness encountered in DLR. The necessary part of the lemma is also not difficult, and a statement and proof (albeit, in a slightly different setting) can be found in Schenone [2010].

Separability (Axiom 5) says that if $p, q \in \mathscr{P}(K \times Y)$ are such that their marginals are identical, ie, $p_{k}=q_{k}$ and $p_{y}=q_{y}$, then $\{p, q\} \sim\{p\}$. This gives us the following lemma.

(27) This is the sense in which the representation in DLR is identified. 
Lemma 18. Let $\left(\mathfrak{U}_{K \times Y}, \mu\right)$ be a finitely additive representation and satisfy Separability (Axiom 5). For $p$ and $q$ that induce the same marginals, ie, $p_{k}=q_{k}$ and $p_{y}=q_{y}, \mu\{\mathfrak{t} \in$ $\left.\mathfrak{U}_{K \times Y}: \mathfrak{u}(p)>\mathfrak{u}(q)\right\}=0$.

Proof. If the lemma were not true, we would have $V(\{p, q\})-V(\{q\})>0$, which contradicts Separability (Axiom 5).

Definition 19. Let $\left(\mathfrak{U}_{K \times Y}, \mu\right)$ be a finitely additive EU representation. The representation is finitely additive, separable if for each $\mathfrak{t}$ in the carrier of $\mu$, there exist $u(\mathfrak{u}) \in \mathcal{U}$ and $v(\mathfrak{u}) \in C(Y)$ such that $\mathfrak{u}(p):=u\left(p_{k} ; \mathfrak{u t}\right)+v\left(p_{y} ; \mathfrak{u t}\right)$ for each $p \in \mathscr{P}(K \times Y)$, and if the mapping $\mathfrak{u} \mapsto(u, v)$ is measurable. A finitely additive, separable representation $\left(\mathfrak{U}_{K \times Y}, \mu\right)$ is additive, separable if $\mu$ is a countably additive probability measure on the Borel sigmaalgebra of $\mathfrak{U}_{K \times Y}$. For ease of notation, we shall suppress the dependence of $u$ and $v$ on $\mathfrak{u t}$.

Lemma 20. A finitely additive EU representation satisfies Separability if, and only if, it is also a finitely additive separable representation as in

$$
V(G)=\int_{\mathfrak{U}_{K \times Y}} \max _{p \in G}\left[u\left(p_{k}\right)+v\left(p_{y}\right)\right] \mathrm{d} \mu(\mathfrak{u})
$$

Proof. The 'if' part is clear. We now prove the 'only if' part. Fix $\tilde{k} \in K$ and $\tilde{y} \in Y$. Since $\frac{1}{2}(k, y)+\frac{1}{2}(\tilde{k}, \tilde{y})$ and $\frac{1}{2}(k, \tilde{y})+\frac{1}{2}(\tilde{k}, y)$ have the same marginals, lemma 18 implies that $\mathfrak{u}\left(\frac{1}{2}(k, y)+\frac{1}{2}(\tilde{k}, \tilde{y})\right)=\mathfrak{t}\left(\frac{1}{2}(k, \tilde{y})+\frac{1}{2}(\tilde{k}, y)\right)$ for all $\mathfrak{t}$ in the carrier of $\mu$. Since each $\mathfrak{t}$ is linear in probabilities, $\mathfrak{t}((k, y))=\mathfrak{t}((k, \tilde{y}))+\mathfrak{t}((\tilde{k}, y))-\mathfrak{t}((\tilde{k}, \tilde{y}))$ must be satisfied for each individual state $\mathfrak{t}$ in the carrier of $\mu{ }^{28}$ Following GP, we define $u(k):=\mathfrak{t}((k, \tilde{y}))$ and $v(y):=\mathfrak{u}((\tilde{k}, y))-\mathfrak{t}((\tilde{k}, \tilde{y}))$ to find $\mathfrak{t}((k, \tilde{y}))=u(k)+v(y)$. This allows us to write $V(G)=$ $\int_{\mathfrak{U}_{K \times Y}} \max _{p \in G}\left[u\left(p_{k}\right)+v\left(p_{y}\right)\right] \mathrm{d} \mu(\mathfrak{u})$ which is the desired separable representation.

\section{D.1. Reduction of the State Space}

Recall that $u:=\left\{u \in \mathbb{R}^{K}: \sum_{i} u_{i}=0\right\}$ is the collection of all vN-M utility functions on $K$ modulo additive constants. It is useful to define the space of all twice-normalized, vN-M utility functions on $K$ as $\mathfrak{U}_{K}:=\left\{r \in \mathcal{U}:\|r\|_{2}=1\right\}$. Since $\mathfrak{U}_{K} \subset \mathcal{U}$, we must have $\sum_{i} r_{i}=0$, so that $r\left(p_{k}^{*}\right)=0$ for all $r \in \mathfrak{U}_{K}$. We now use lemma 17 to show that for any finitely additive separable representation, the state space can be viewed as (a subset of) $\mathfrak{U}_{K} \times[0,1] \times \mathfrak{U}_{Y}$.

Proposition 21. A preference $\succsim$ over the compact subsets of $\mathscr{P}(K \times Y)$ with a finitely additive representation $\left(\mathfrak{U}_{K \times Y}, \mu\right)$ satisfies Separability (Axiom 5 ) if, and only if, there is a change of state space as in lemma 17 that allows to write a finitely additive representation of $\succsim$ of the form

$$
\int_{\mathfrak{U}_{K} \times[0,1] \times \mathfrak{U}_{Y}} \max _{p \in G}\left[\gamma r\left(p_{k}\right)+(1-\gamma) v\left(p_{y}\right)\right] \mathrm{d} \mu^{\prime}(r, \gamma, v)
$$

where $\mu^{\prime}$ is a charge on $\mathfrak{U}_{K} \times[0,1] \times \mathfrak{U}_{Y}$.

(28) In comparison, the weaker separability axiom in GP would only imply this condition for the preference functional $V$ that aggregates over all states. 
Proof. The 'if' part is immediate, so we only prove the 'only if' part of the proposition. Let $\left(\mathfrak{U}_{K \times Y}, \mu\right)$ be a finitely additive representation. (Recall that Theorem 4 guarantees the existence of a finitely additive representation, though we have not ruled out the possibility that there could be many such representations.) By lemma 20, every such representation must have the property that for every $\mathfrak{t}$ in the carrier of $\mu, \mathfrak{t}(p)=u\left(p_{k}\right)+v\left(p_{y}\right)$, where $u \in \mathcal{U}$ and $v \in C(Y)$ are $\mathrm{vN}-\mathrm{M}$ functions and the mapping $\mathfrak{t} \mapsto(u, v)$ is measurable.

Every separable utility function $u\left(p_{k}\right)+v\left(p_{y}\right)$ is of the form $\alpha r\left(p_{k}\right)+\beta w\left(p_{y}\right)$, where $r \in \mathfrak{U}_{K}, w \in \mathfrak{U}_{Y}$, and $(\alpha, \beta) \in \mathbb{R}_{+}^{2} \backslash(0,0)$. Let $X:=\left(\mathfrak{U}_{K} \times \mathbb{R}_{+}\right) \times\left(\mathfrak{U}_{Y} \times \mathbb{R}_{+}\right)$, and consider $\mathfrak{U}_{K \times Y} \cap X$. A finitely additive separable representation is one where $\mu\left(\mathfrak{U}_{K \times Y} \cap X\right)=1$.

An even smaller state space is $\mathfrak{U}_{K} \times[0,1] \times \mathfrak{U}_{Y}$, wherein the utility in state $(r, \gamma, v)$ is $\gamma r\left(p_{k}\right)+(1-\gamma) w\left(p_{y}\right)$.

Define $\Psi: \mathfrak{U}_{K \times Y} \cap X \rightarrow \mathfrak{U}_{K} \times[0,1] \times \mathfrak{U}_{Y}$ as follows: $\Psi:((r, \alpha),(w, \beta)) \mapsto$ $(r, \alpha /(\alpha+\beta), w)$. It is clear that $\Psi$ is continuous, and hence measurable. Moreover, $\Psi$ is also a bijection. To see this, suppose there are $\alpha, \alpha^{\prime}, \beta, \beta^{\prime}$ such that $\alpha /(\alpha+\beta)=\alpha^{\prime} /\left(\alpha^{\prime}+\beta^{\prime}\right)$. This implies $\alpha^{\prime}=\rho \alpha$ and $\beta^{\prime}=\rho \beta$ for some $\rho>0$, which is impossible since, as mentioned above, $\mathfrak{U}_{K \times Y} \cap X$ only contains functions that are unique up to scaling.

By lemma 17, we may define the charge $\mu^{\prime}(D):=\mu\left(\Psi^{-1} D\right)$ on $\mathfrak{U}_{K} \times[0,1] \times \mathfrak{U}_{Y}$, and restrict attention to a separable representation on this state space.

In sum, a finitely additive, separable, EU representation of $\succsim$ is given by a charge on $\mathfrak{U}_{K} \times[0,1] \times \mathfrak{U}_{Y}$, with a typical vN-M utility function of the form $\gamma r\left(p_{k}\right)+(1-\gamma) w\left(p_{y}\right){ }^{29}$ Notice that since $\Psi$ is continuous, the charge $\mu^{\prime}$ is normal (ie, both inner and outer regular).

\section{D.2. Additively Separable Representation}

We now show that in the presence of some additional assumptions, the normal probability charge $\mu^{\prime}$ in proposition 21 can be replaced by a regular probability measure, leading to an additive, separable EU representation. Recall that $\mu^{\prime}$ is a regular probability measure if it is (i) regular (ie, outer regular and tight), and (ii) a probability measure, ie, is countably additive, and has $\mu^{\prime}\left(\mathfrak{U}_{K} \times[0,1] \times \mathfrak{U}_{Y}\right)=1$, and is defined on the Borel sigma-algebra of $\mathfrak{U}_{K} \times[0,1] \times \mathfrak{U}_{Y}$.

Definition 22. Let $\left(\mathfrak{U}_{K} \times[0,1] \times \mathfrak{U}_{Y}, \mu^{\prime}\right)$ be a finitely additive separable EU representation. The representation is $\boldsymbol{Y}$-simple if the marginal charge of $\mu^{\prime}$ on $\mathfrak{U}_{Y}$ - given by $\int_{\mathfrak{U}_{K} \times[0,1] \times D} \mathrm{~d} \mu(r, \gamma, w)$ for any $D \in \mathscr{A}_{\mathfrak{U}_{Y}}$ - has a finite carrier. It is $\boldsymbol{Y}$-trivial if the carrier of the marginal on $\mathfrak{U}_{Y}$ is a singleton.

Lemma 23. Every finitely additive, separable representation that is $Y$-simple can be extended uniquely to an additive, separable, $Y$-simple representation

$$
V(G)=\int_{\mathfrak{U}_{K} \times[0,1] \times\left\{w_{1}, \ldots, w_{n}\right\}} \max _{p \in G}\left[\gamma r\left(p_{k}\right)+(1-\gamma) w\left(p_{y}\right)\right] \mathrm{d} \mu(r, \gamma, w)
$$

(29) In our terms, the representation of HHT is a finitely additive, separable, EU representation, where there is no uncertainty about $r \in \mathfrak{U}_{K}$ or $v \in \mathfrak{U}_{Y}$, so that all the uncertainty is about the stochastic discount factor $\gamma \in[0,1]$. 
Proof. As $\mu^{\prime}$ is $Y$-simple, there must exist an $n \in \mathbb{N}$ with $n>0$ such that the carrier of $\mu^{\prime}$ is a closed subset of $\mathfrak{U}_{K} \times[0,1] \times\left\{w_{1}, \ldots, w_{n}\right\}$, where $\left\{w_{1}, \ldots, w_{n}\right\} \subset \mathfrak{U}_{Y}$. Since $\mathfrak{U}_{K} \times[0,1] \times\left\{w_{1}, \ldots, w_{n}\right\}$ is compact, it follows immediately that the carrier of $\mu^{\prime}$ is also compact.

The charge $\mu^{\prime}$ is normal (see footnote 23), so for any Borel (algebra) measurable $A \subset \mathfrak{U}_{K} \times[0,1] \times\left\{w_{1}, \ldots, w_{n}\right\}$, the inner regularity of $\mu^{\prime}$ implies that $\mu^{\prime}(A)=\sup \{\mu(C)$ : $C \subset A$ and closed $\}$. Since $\mathfrak{U}_{K} \times[0,1] \times\left\{w_{1}, \ldots, w_{n}\right\}$ is compact, it follows that any closed $C \subset A$ is also compact. Therefore, the charge $\mu^{\prime}$ is 'tight' relative to a compact class of sets, namely the collection of all compact subsets of $\mathfrak{U}_{K} \times[0,1] \times\left\{w_{1}, \ldots, w_{n}\right\}$. By Theorem 9.12 of Aliprantis and Border [1999], $\mu^{\prime}$ is also countably additive (on the algebra of open sets).

By the Carathéodory Extension Procedure Theorem, $\mu^{\prime}$ can be uniquely extended from the algebra of open sets to the Borel sigma-algebra of $\mathfrak{U}_{K} \times[0,1] \times\left\{w_{1}, \ldots, w_{n}\right\}$. (The Carathéodory Theorem is Theorem 9.22 of Aliprantis and Border [1999]. The extension of the measure is unique since $\mu^{\prime}$ is a finite measure.) The unique extension of $\mu^{\prime}$ will be written as $\mu$.

Finally, Theorem 10.7 of Aliprantis and Border [1999] says that a measure on a Polish space is regular, and because $\mathfrak{U}_{K} \times[0,1] \times\left\{w_{1}, \ldots, w_{n}\right\}$ is a compact subset of Euclidean space, it follows that $\mu$ is regular.

\section{D.3. Identification of the Representation}

Recall that in the original, abstract EU representation theorem, Theorem 4 , we are unable to establish that $\mu$ is a regular measure or that the representation is jointly identified. We have seen that in the presence of additional assumptions, it is possible to show that $\mu$ is a regular measure. We now show that in that case, the representation can be jointly identified.

Proposition 24. Suppose a continuous preference $\succsim$ has an additive, separable, $Y$-simple representation as in equation (D.2). Then, the representation is jointly identified, ie, the measure $\mu$ is unique given the state space $\mathfrak{U}_{K} \times[0,1] \times\left\{w_{1}, \ldots, w_{n}\right\}$.

Proof. Let $\succsim$ have a utility representation $V$ as in (D.2), and let $\mu_{0}=\mu$. Suppose there is another regular measure $\mu_{1}$ (that need not be a probability measure) on $\mathfrak{U}_{K} \times[0,1] \times$ $\left\{w_{n+1}, \ldots, w_{n+m}\right\}$ such that

$$
V(G)=\int_{\mathfrak{U}_{K} \times[0,1] \times\left\{w_{n+1}, \ldots, w_{n+m}\right\}} \max _{p \in G}\left[\gamma r\left(p_{k}\right)+(1-\gamma) w\left(p_{y}\right)\right] \mathrm{d} \mu_{1}(r, \gamma, w)
$$

It is without loss of generality to consider $\mu_{0}$ and $\mu_{1}$ as measures on $\mathfrak{U}_{K} \times[0,1] \times\left\{w_{1}, \ldots, w_{n+m}\right\}$. We show that $\mu_{1}=\mu_{0}$.

Let $Y_{0} \subset Y$ be a finite set such that (i) $w_{i}$ is nonconstant on $Y_{0}$ for all $i=1, \ldots, n$, and (ii) for each $i, j \in 1, \ldots, n$ where $i \neq j$, there exists $y_{i j} \in Y_{0}$ such that $w_{i}\left(y_{i j}\right) \neq w_{j}\left(y_{i j}\right)$. Since each $w_{i}$ is nonconstant on $Y$, the first requirement is easily satisfied. The second requirement is also satisfied, since $w_{i}$ is a continuous function on $Y$ and $i \neq j$ implies $w_{i} \neq w_{j}$, which in turn implies that the two functions must disagree somewhere. 
Now consider the set $B:=K \times Y_{0}$, and the domain $\mathscr{F}(\mathscr{P}(B))$. Then, each measure $\mu_{j}, j=0,1$, induces the preference functional $W_{j}$ on $\mathscr{F}(\mathscr{P}(B))$ as follows:

$$
W_{j}(G)=\int_{\mathfrak{U}_{K} \times[0,1] \times\left\{w_{1}, \ldots, w_{n}\right\}} \max _{p \in G}\left[\gamma r\left(p_{k}\right)+(1-\gamma) w\left(p_{y}\right)\right] \mathrm{d} \mu_{j}(r, \gamma, w)
$$

Define, as in DLR, $\mathfrak{U}_{B}:=\left\{r \in \mathbb{R}^{B}: \sum_{i} r_{i}=0, \sum r_{i}^{2}=1\right\}$. In that case, $\mathfrak{U}_{K} \times$ $[0,1] \times\left\{w_{1}, \ldots, w_{n}\right\}$ is isomorphic to a subset of $\mathfrak{U}_{B}$. Indeed, for any vN-M utility function on $K \times Y_{0}$ of the form $\alpha r\left(q_{k}\right)+(1-\alpha) w\left(q_{y}\right)$, the two normalizations that map the function into $\mathfrak{U}_{B}$ are continuous.

Thus, $\mu_{0}$ and $\mu_{1}$ are transformed into measures on $\mathfrak{U}_{B}$ in the obvious way, and have supports on sets (in $\mathfrak{U}_{B}$ ) that are isomorphic to $\mathfrak{U}_{K} \times[0,1] \times\left\{w_{1}, \ldots, w_{n}\right\}$. From the definition of the functionals $W_{0}$ and $W_{1}$, we know that for each menu $G \in \mathscr{F}(\mathscr{P}(B))$, we have $W_{0}(G)=W_{1}(G)$. The uniqueness part of the additive EU representation theorem in DLR now says that the two transformed measures agree on $\mathfrak{U}_{B}$, and hence $\mu_{0}=\mu_{1}$, as desired.

It is useful to write $Y$-trivial, additive, separable EU representations of a $\succsim$ by the collection $(\mathcal{U}, v, \mu)$ where $v: Y \rightarrow \mathbb{R}$ is a vN-M function, and $\mu$ is a probability measure on (the Borel sigma-algebra of) $\mathcal{U}$. It induces the preference functional

$$
V(G)=\int_{u} \max _{p \in G}\left[u\left(p_{k}\right)+v\left(p_{y}\right)\right] \mathrm{d} \mu(u)
$$

that represents the preference $\succsim$ over menus. The transformation from the state space with $(r, \alpha) \in \mathfrak{U}_{K} \times[0,1]$ to the state space with $u \in U$ is achieved by setting $u:=\frac{\alpha}{(1-\alpha)} r$ and by applying the appropriate transformation to the measure, as in lemma 17.

\section{E. Proofs from Section 4.2}

\section{E.1. Proof of Theorem 2}

In section E.1.1, we establish that for each $s \in S$, the preference $\succsim_{s}$ has a $H$-trivial, separable, additive representation as in (D.3). This amounts to showing that the relevant state space is isomorphic to $U$. In section E.1.2, we show that the parameter of the representation of $\succsim_{s}$, namely the measure $\mu_{s}$ on $\mathcal{U}$, is uniquely identified up to a scaling. In section E.1.3, we show that $\succsim$ has a recursive representation, possibly with state-dependent discount factors. In section E.1.4. we show that there exists an equivalent representation of $\succsim$ with a constant discount factor. We also show that the collection of measures $\left(\mu_{s}\right)$ is unique up to a common scaling, and that the Markov chain on $S$ with transition probabilities $\Pi$ has the unique invariant distribution $\pi_{0}$.

\section{E.1.1. Separable Representation}

It is easy to see that $\succsim_{s}$ is continuous, satisfies Independence, Monotonicity, and Separability, so that by proposition 21 . $\succsim_{s}$ has a finitely additive separable representation. We shall now show that the representation is also $H$-trivial. 
Proposition 25. Suppose $\succsim_{s}$ has a finitely additive separable representation. If $\succsim_{s}$ also satisfies State Contingent CSR (Axiom X8), then the representation is $H$-trivial.

Proof. As in Proposition 21, we know that a separable, finitely additive separable representation has the form

$$
U_{S}(x)=\int_{\mathfrak{U}_{K} \times[0,1] \times \mathfrak{U}_{H}} \max _{p \in x}\left[\gamma r\left(p_{k}\right)+(1-\gamma) v\left(p_{h}\right)\right] \mathrm{d} \mu_{s}(r, \gamma, v)
$$

For an arbitrary consumption prize $k \in K$, let $(k, A)$ be a rectangular menu, ie, a menu defined as $(k, A):=\left\{\left(k, p_{z}\right): p_{z} \in A, A \in \mathscr{F}_{H}\right\}$. Define a utility function $W_{s}: \mathscr{F}_{H} \rightarrow \mathbb{R}$ as $W_{s}(A)=U_{s}(k, A)$. It follows from the separability of the representation that the choice of $k \in K$ only affects $W_{s}$ up to a constant. Let $\mathrm{d} \mu_{s}^{*}(v)=\iint_{\mathfrak{L}_{K} \times[0,1]} \mathrm{d} \mu_{s}(r, \gamma, v)$ be the marginal of $\mu$ on $\mathfrak{U}_{H}$. Then, $W_{S}(A)=\int_{\mathfrak{U}_{H}} \max _{p_{h} \in A} v\left(p_{h}\right) \mathrm{d} \mu_{s}^{*}(v)+$ constant.

By proposition 16 above, State Contingent CSR (Axiom X8) implies that $W_{s}(A)=$ $\max _{p_{h} \in A} v_{s}\left(p_{h}\right)$, where $v_{s} \in C(H)$ is given by $v_{s}(f)=W_{s}(\{f\})$. But this implies that $\max _{p_{h} \in A} v_{s}\left(p_{h}\right)=\int \max _{p_{h} \in A} v\left(p_{h}\right) \mathrm{d} \mu^{*}(v)$. Therefore, the carrier of $\mu^{*}$ must be a singleton.

It follows from lemma 23 that $\mu_{s}$ can be extended to a measure in a unique way. Thus, as mentioned at the end of section D.3. $\succsim_{s}$ has a separable, additive, $H$-trivial EU representation that, after a change of the state space, can be written as

$$
U_{s}(x)=\int_{U} \max _{p \in x}\left[u\left(p_{k}\right)+v_{s}\left(p_{h}\right)\right] \mathrm{d} \mu_{s}(u)
$$

where, abusing notation, the transformed measure on $U$ is also denoted by $\mu_{s}$. We end with the observation that $\mu_{s}$ is nice. To see this, note that there exists $p_{h}^{*} \in \mathscr{P}(H)$ such that $v_{s}\left(p_{h}^{*}\right)=0$. Now consider the menu $\left(k, p_{h}^{*}\right)$. It is easy to see that $U_{s}\left(\left(k, p_{h}^{*}\right)\right)=\mu_{s} u(k):=$ $\int_{u} u(k) \mathrm{d} \mu_{s}(u)$. But $U_{s}\left(\left(k, p_{h}^{*}\right)\right)$ is finite, which implies $\mu_{s} u(k)$ is finite for every $k \in K$, which proves that $\mu_{s}$ is nice.

The preference $\succsim$ on $H$ is continuous and satisfies Independence (Axiom X3). Therefore, there exists a representation $W(f):=\sum_{s} \pi_{0}(s) U_{s}(f(s))$ of $\succsim$, where $\pi_{0} \in \mathscr{P}(S)$.

\section{E.1.2. Identification}

Section E.1.1 shows that the state space relevant for a finitely additive, separable, $H$-trivial representation is $\mathcal{U}$. Our goal in this section is to show that the measure $\mu_{s}$ on $\mathcal{U}$ and $v_{s}$ are unique up to a common scaling. We shall say that $v_{s}$ is non-degenerate if $v_{s} \neq \mathbf{0}$, ie, if $v_{s}$ is not identically zero.

Proposition 26. Consider two separable, $H$-trivial, additive EU representations, $\left(v_{s}, \mu_{s}\right)$ and $\left(v_{s}^{\prime}, \mu_{s}^{\prime}\right)$, of $\succsim_{s}$, wherein both $v_{s}$ and $v_{s}^{\prime}$ are non-degenerate. There exists $\zeta>0$ such that the following properties hold:

(a) $\mu_{s}(\zeta D)=\mu_{s}^{\prime}(D)$ for all measurable $D \subset \mathcal{U}$,

(b) $v_{s}^{\prime}=\zeta v_{s}+$ constant. 
Proof. By lemma 17, our result on the change of state space, we know that there exists a measurable bijection $\Psi: \mathcal{U} \rightarrow \mathcal{U}$, and integrable functions $(\zeta, \xi): \mathcal{U} \mapsto\left(\mathbb{R}_{++}, \mathbb{R}\right)$ such that for each $u^{\prime}$ in the support of $\mu_{s}^{\prime}$, we have $u^{\prime}\left(p_{k}\right)+v_{s}^{\prime}\left(p_{h}\right)=\zeta(u)\left[(\Psi u)\left(p_{k}\right)+v_{s}\left(p_{h}\right)\right]+\xi(u)$.

Consider two lotteries $p, q$ such that $p_{k}=q_{k}$ but where $v_{s}\left(p_{h}\right) \neq v_{s}\left(q_{h}\right)$. Then,

$$
\begin{aligned}
{\left[u^{\prime}\left(p_{k}\right)+v_{s}^{\prime}\left(p_{h}\right)\right]-\left[u^{\prime}\left(q_{k}\right)+v_{s}^{\prime}\left(q_{h}\right)\right] } & =v_{s}^{\prime}\left(p_{h}\right)-v_{s}^{\prime}\left(q_{h}\right) \\
& =\zeta(u)\left[v_{s}\left(p_{h}\right)-v_{s}\left(q_{h}\right)\right]
\end{aligned}
$$

But this implies $\zeta(u)=\frac{v_{s}^{\prime}\left(p_{h}\right)-v_{s}^{\prime}\left(q_{h}\right)}{v_{s}\left(p_{h}\right)-v_{s}\left(q_{h}\right)}$ for all $u$, and hence $\zeta(u)$ is constant, which proves (b).

Let $V_{s}^{\prime}$ be the functional induced by $\left(v_{s}^{\prime}, \mu_{s}^{\prime}\right)$. Then,

$$
\begin{aligned}
V_{s}^{\prime}(x) & =\int_{u} \max _{p \in x}\left[u^{\prime}\left(p_{k}\right)+v_{s}^{\prime}\left(p_{h}\right)\right] \mathrm{d} \mu_{s}^{\prime}\left(u^{\prime}\right) \\
& =\int_{u} \max _{p \in x} \zeta\left[(\Psi u)\left(p_{k}\right)+v_{s}\left(p_{h}\right)\right] \mathrm{d} \tilde{\mu}_{s}^{\prime}(u)+\int \xi \mu_{s}^{\prime} \\
& =\zeta \int_{u} \max _{p \in x}\left[\tilde{u}\left(p_{k}\right)+v_{s}\left(p_{h}\right)\right] \mathrm{d} \mu_{s}^{\prime \prime}(\tilde{u})+\text { constant }
\end{aligned}
$$

where $\tilde{\mu}_{s}^{\prime}$ and $\mu_{s}^{\prime \prime}$ each obtain from a change of state space. Proposition 24 says that $\mu_{s}^{\prime \prime}=\mu_{s}$, which implies that $\Psi$ is the identity mapping. Thus, it must be that $\mu_{s}^{\prime}(D)=\mu_{s}(\zeta D)$ for all measurable $D \subset \mathcal{~}$. These observations prove part (a).

\section{E.1.3. Recursive Representation}

Thus far, we have established that $\succsim$ has a separable representation of the form

$$
W\left(f, \pi_{0}\right)=\sum_{s} \pi_{0}(s)\left[\int_{u} \max _{p \in f(s)}\left[u\left(p_{k}\right)+v_{s}\left(p_{h}\right)\right] \mathrm{d} \mu_{s}(u)\right]
$$

In the representation above, each $v_{s}: H \rightarrow \mathbb{R}$ is continuous (recall that $H$ is a convex set). Let $\succsim_{s}^{*}$ be the preference relation on $H$ induced by $v_{s}$.

Proposition 27. Suppose $\succsim$ has a representation as in $(\overline{E .2})$ and satisfies Singleton Indifference to Timing (Axiom X7), Then, each $v_{s}: H \rightarrow \mathbb{R}$ is linear, ie, $\succsim_{s}^{*}$ satisfies Independence.

Proof. As above, we have $U_{s}: \mathscr{F} \rightarrow \mathbb{R}$ given by $U_{s}(x)=\int_{U} \max _{p \in x}\left[u\left(p_{k}\right)+v_{s}\left(p_{h}\right)\right] \mathrm{d} \mu_{s}(u)$, and $U_{s}$ represents $\succsim_{s}$. Let $f, g \in H$ such that $v_{s}(f)>v_{s}(g)$ which implies $U_{s}\left(\left\{p_{k}^{*}, f\right\}\right)>$ $U_{s}\left(\left\{p_{k}^{*}, g\right\}\right)$. Let $f^{\prime} \in H$, and fix $\lambda \in(0,1]$. By the linearity of $U_{s}$, we have $U_{s}\left(\lambda\left\{p_{k}^{*}, f\right\}+\right.$ $\left.(1-\lambda)\left\{p_{k}^{*}, f^{\prime}\right\}\right)>U_{s}\left(\lambda\left\{p_{k}^{*}, g\right\}+(1-\lambda)\left\{p_{k}^{*}, f^{\prime}\right\}\right)$. But $\succsim_{s}$ satisfies Singleton Indif ference to Timing (Axiom X7), which implies that $U_{s}\left(\lambda\left\{p_{k}^{*}, f\right\}+(1-\lambda)\left\{p_{k}^{*}, f^{\prime}\right\}\right)=$ $U_{s}\left(\left\{p_{k}^{*}, \lambda f+(1-\lambda) f^{\prime}\right\}\right)$, so that $U_{s}\left(\left\{p_{k}^{*}, \lambda f+(1-\lambda) f^{\prime}\right\}\right)>U_{s}\left(\left\{p_{k}^{*}, \lambda g+(1-\lambda) f^{\prime}\right\}\right)$, which in turn implies that $v_{s}\left(\lambda f+(1-\lambda) f^{\prime}\right)>v_{s}\left(\lambda g+(1-\lambda) f^{\prime}\right)$. We have just established that $\succsim_{s}^{*}$ also satisfies Independence (Axiom X3), from which it follows that $v_{s}$ is linear, as desired.

Proposition 28. Suppose $\succsim$ has a representation as in (E.2). If $\succsim$ satisfies Statewise Nontriviality (Axiom X1), History Independence (Axiom X9), and Aggregate Stationarity (Axiom X6), then each $\succsim_{s}^{*}$ also satisfies Monotonicity and Statewise Non-triviality (Axiom X1). 
Proof. As $v_{s}$ is linear and continuous, it follows that there exist utility functions $w_{s, s^{\prime}}: \mathscr{F} \rightarrow$ $\mathbb{R}$ for all $s^{\prime} \in S$ such that $v_{s}(f)=\sum_{s^{\prime}} w_{s, s^{\prime}}\left(f\left(s^{\prime}\right)\right)$. Notice that by History Independence (Axiom X9), we may assume that $w_{s, s^{\prime}}=\pi_{s}\left(s^{\prime}\right) w_{s^{\prime}}$ for all $s, s^{\prime} \in S$, where $\pi_{s}\left(s^{\prime}\right)>0$.

Notice that $f_{s}^{x} \succsim f_{s}^{y}$ if, and only if, $U_{s}(x) \geqslant U_{s}(y)$. By Aggregate Stationarity (Axiom X6), this is equivalent to requiring that $\left\{\left(k, f_{s}^{x}\right)\right\} \succsim\left\{\left(k, f_{s}^{y}\right)\right\}$, which holds if, and only if, $\sum_{s}^{\prime} \pi_{0}\left(s^{\prime}\right) v_{s^{\prime}}\left(f_{s}^{x}\right) \geqslant \sum_{s}^{\prime} \pi_{0}\left(s^{\prime}\right) v_{s^{\prime}}\left(f_{s}^{y}\right)$. By the observations above, this is equivalent to $\sum_{s^{\prime}} \pi_{0}\left(s^{\prime}\right) \pi_{s^{\prime}}(s) w_{s}(x) \geqslant \sum_{s^{\prime}} \pi_{0}\left(s^{\prime}\right) \pi_{s^{\prime}}(s) w_{s}(y)$. Let $W_{s}^{\prime}(x):=\sum_{s^{\prime}} \pi_{0}\left(s^{\prime}\right) \pi_{s^{\prime}}(s) w_{s}(x)$. Then, $W_{s}^{\prime}$ is linear on $\mathscr{F}$ and $W_{s}^{\prime}(x) \geqslant W_{s}^{\prime}(y)$ if, and only if, $U_{s}(x) \geqslant U_{s}(y)$.

Thus, $\succsim_{s}^{*}$ satisfies Monotonicity (Axiom X4) if, and only if, $w_{s}(x \cup y) \geqslant w_{s}(x)$ for all $x, y \in \mathscr{F}$. But $w_{s}$ is monotone with respect to set inclusion if, and only if, $U_{s}$ is, which proves that $f_{s^{\prime}}^{x \cup y} \succsim_{s}^{*} f_{s^{\prime}}^{x}$ because $x \cup y \succsim_{s} x$.

Finally, to see that $\succsim_{s}^{*}$ satisfies Statewise Non-triviality (Axiom X1), notice that $W_{\tilde{s}}^{\prime}=\sum_{s} \pi_{0}(s) \pi_{s}(\tilde{s}) w_{\tilde{s}}$. But $W_{\tilde{s}}^{\prime}$ is non-trivial because $\succsim_{\tilde{s}}$ is non-trivial. Therefore, it must be that $w_{\tilde{s}}$ is non-trivial, from which it follows that $\succsim_{\widetilde{s}}^{*}$ satisfies Statewise Non-triviality (Axiom X1).

We now establish the existence of a recursive representation.

Proposition 29. Let $\succsim$ have a representation as in (E.2), and suppose $\succsim$ satisfies Statewise Non-triviality (Axiom X1), History Independence (Axiom X9), Aggregate Stationarity (Axiom X6), and Singleton Indifference to Timing (Axiom X7), Then, there is a value function

$$
V\left(f, s^{\prime}\right)=\sum_{s} \Pi\left(s^{\prime}, s\right)\left[\int_{u} \max _{p \in f(s)}\left[u\left(p_{k}\right)+\delta_{s} V\left(p_{h}, s\right)\right] \mathrm{d} \mu_{s}(u)\right]
$$

where $\Pi$ governs transition probabilities for a Markov process on $S$ and $\Pi\left(s^{\prime}, s\right)>0$ for all $s^{\prime}, s \in S$, such that

$$
V\left(f, \pi_{0}\right)=\sum_{s} \pi_{0}(s)\left[\int_{u} \max _{p \in f(s)}\left[u\left(p_{k}\right)+\delta_{s} V\left(p_{h}, s\right)\right] \mathrm{d} \mu_{s}(u)\right]
$$

represents $\succsim$.

Proof. Fix $s^{\prime} \in S$ and consider the act $f_{s^{\prime}}^{x}$. Then,

$$
W\left(f_{s^{\prime}}^{x}, \pi_{0}\right)=\pi_{0}\left(s^{\prime}\right) \int_{u} \max _{p \in x}\left[u\left(p_{k}\right)+v_{s^{\prime}}\left(p_{h}\right)\right] \mathrm{d} \mu_{s^{\prime}}(u)+(\cdot)
$$

As noted above, $v_{s}(\cdot)$ induces a preference $\succsim_{s}^{*}$ on $H$ such that (i) $\succsim_{s}^{*}$ is continuous, (ii) $\succsim_{s}^{*}$ satisfies Independence (proposition 27), and (iii) $\succsim_{s}^{*}$ satisfies Monotonicity (proposition 28. Then, by the Mixture Space Theorem and by Theorem 4, $v_{s}$ has a (state-separabale) finitely additive representation, which can be written as

$$
v_{s}\left(f_{s^{\prime}}^{x}\right)=\delta_{s} \pi_{s}\left(s^{\prime}\right) \int_{\mathfrak{U}_{K \times H}} \max _{p \in x} \mathfrak{t}(p) \mathrm{d} \tilde{\mu}_{S^{\prime}}^{s}(\mathfrak{u})+\delta_{s} \sum_{t \neq s^{\prime}} \pi_{s}(t) \int_{\mathfrak{U}_{K \times H}} \max _{p \in f(t)} \mathfrak{t}(p) \mathrm{d} \tilde{\mu}_{t}^{s}(\mathfrak{u})
$$

where $\delta_{s}$ is a scaling factor chosen so that (i) $\pi_{s} \in \mathscr{P}(S)$ is strictly positive and (ii) $\tilde{\mu}_{t}^{s}$ is a probability charge for all $t \in S$. Such a choice can be made as follows: If $\tilde{\mu}_{t}^{s}\left(\mathfrak{U}_{K \times H}\right)>1$, 
define $\pi_{s}^{\prime}(t):=\tilde{\mu}_{t}^{s}\left(\mathfrak{U}_{K \times H}\right)$, so that we can take $\tilde{\mu}_{t}^{s}$ to be a probability measure. Because $\pi_{s}^{\prime}(t)>0$ for all $t \in S$, it follows that we may let $\delta_{s}=\sum_{t} \pi_{s}^{\prime}(t)$, so that the scaling factor $\pi_{s}^{\prime}(t)$ can be replaced by $\pi_{s}(t):=\pi_{s}^{\prime}(t) / \delta_{s}$.

Singleton Indifference to Timing (Axiom X7) implies that $\pi_{0}\left(s^{\prime}\right) \int_{U} \max _{p \in x}\left[u\left(p_{k}\right)+\right.$ $\left.v_{s^{\prime}}\left(p_{h}\right)\right] \mathrm{d} \mu_{s^{\prime}}(u)$ and $\delta_{s} \pi_{s}\left(s^{\prime}\right) \int_{\mathfrak{U}_{K \times H}} \max _{p \in x} \mathfrak{u}(p) \mathrm{d} \tilde{\mu}_{s^{\prime}}^{s}(\mathfrak{u})$ are (positive) affine transformations of each other. By Statewise Non-triviality (Axiom X1) every state is non-null under $\pi_{0}$, and by History Independence (Axiom X9], every state is also non-null under $\pi_{s}$, ie, $\pi_{s}\left(s^{\prime}\right)>0$ (this was established in proposition 28). Hence the measures $\left\{\pi_{0}, \pi_{s}: s \in S\right\}$ have full support.

Recall that $\mu_{s^{\prime}}$ defined on $\mathcal{U}$ is the marginal of a measure on $\mathcal{U} \times \mathfrak{U}_{H}$, where the marginal on $\mathfrak{U}_{H}$ has a singleton carrier. The transformations of the state space used in obtaining a separable representation are an instance of those considered in lemma 17, so that we may regard $\mu_{S^{\prime}}$ as a measure on $\mathfrak{U}_{K \times H}$.

Since both $\tilde{\mu}_{s^{\prime}}^{s}$ and $\mu_{s^{\prime}}$ are probability charges, we must have $\tilde{\mu}_{s^{\prime}}^{s}=\mu_{s^{\prime}}$ for all $s \in S$. Therefore, each $v_{s}$ can be written as

$$
v_{s}(f)=\delta_{s} \sum_{s^{\prime}} \pi_{s}\left(s^{\prime}\right) \int_{u} \max _{p \in f\left(s^{\prime}\right)}\left[u\left(p_{k}\right)+v_{s^{\prime}}\left(p_{h}\right)\right] \mathrm{d} \mu_{s^{\prime}}(u)
$$

Define the Markov transition probabilities on $S$ to be $\Pi\left(s, s^{\prime}\right):=\pi_{s}\left(s^{\prime}\right)$, and let $V(f, s):=$ $v_{s}(f) / \delta_{s}$ so that

$$
V(f, s)=\sum_{s^{\prime}} \Pi\left(s, s^{\prime}\right) \int_{u} \max _{p \in f\left(s^{\prime}\right)}\left[u\left(p_{k}\right)+\delta_{s^{\prime}} V\left(p_{h}, s^{\prime}\right)\right] \mathrm{d} \mu_{s^{\prime}}(u)
$$

Finally, define

$$
V\left(f, \pi_{0}\right):=W\left(f, \pi_{0}\right)=\sum_{s} \pi_{0}(s) \int_{u} \max _{p \in f(s)}\left[u\left(p_{k}\right)+\delta_{s} V\left(p_{h}, \pi_{s}\right)\right] \mathrm{d} \mu_{s}(u)
$$

to attain the desired recursive representation.

\section{E.1.4. Representation with a Constant Discount Factor - Existence and Uniqueness}

Proposition 29 establishes the existence of a recursive value function as in (E.3), which can be described by the parameters $\left(\left(\mu_{s}, \pi_{s}, \delta_{s}\right)_{s \in S}\right)$. We will now show that there exists an equivalent representation with a constant discount factor. Let $\xi \in \mathbb{R}_{++}^{S}$, and let $\langle\pi, \xi\rangle:=$ $\left.\sum_{s} \pi(s) \xi(s)\right)$ for any $\pi \in \mathscr{P}(S)$.

Proposition 30. Let $\left(\left(\mu_{s}, \pi_{s}, \delta_{s}\right)_{s \in S}\right)$ and $\left(\left(\hat{\mu}_{s}, \hat{\pi}_{s}, \hat{\delta}_{s}\right)_{s \in S}\right)$ be two recursive representations of $\succsim$ as in E.3. Then, there exists $\xi \in \mathbb{R}_{++}^{S}$ such that the parameters are related by the following transformations.

- $\hat{\pi}_{0}(s):=\pi_{0}(s) \xi(s) /\left\langle\pi_{0}, \xi\right\rangle$

- $\hat{\pi}_{s}\left(s^{\prime}\right):=\pi_{s}\left(s^{\prime}\right) \xi\left(s^{\prime}\right) /\left\langle\pi_{s}, \xi\right\rangle$

- $\hat{\mu}(D):=\mu(D / \xi(s))$ 
- $\hat{\delta}_{s}:=\delta_{s}\left\langle\pi_{s}, \xi\right\rangle / \xi(s)$

- $\hat{V}\left(\cdot, \hat{\pi}_{s}\right):=V\left(\cdot, \pi_{s}\right) /\left\langle\pi_{s}, \xi\right\rangle$

Moreover, if $\left(\left(\mu_{s}, \pi_{s}, \delta_{s}\right)_{s \in S}\right)$ is a representation of $\succsim$, then the transformed set of parameters $\left(\left(\hat{\mu}_{s}, \hat{\pi}_{s}, \hat{\delta}_{s}\right)_{s \in S}\right)$ also represents $\succsim$.

Proof. The last part of the proposition is immediate. By our identification result, proposition 26. there exists $\xi \in \mathbb{R}_{++}^{S}$ such that (i) $\hat{\mu}_{s}(D)=\mu_{s}(D / \xi(s))$ for all $s \in S$ and for all Borel measurable $D \subset \mathcal{U}$, and (ii) $\hat{\delta}_{s} \hat{V}(\cdot, s):=\delta_{s} V(\cdot, s) / \xi(s)$. By construction, $\hat{V}(\cdot, s)=$ $V(\cdot, s) /\left\langle\pi_{s}, \xi\right\rangle$, and hence we must have $\hat{\delta}_{s}:=\delta_{s}\left\langle\pi_{s}, \xi\right\rangle / \xi(s)$. Finally, the identification from the Mixture Space Theorem implies that $\hat{\pi}_{s}\left(s^{\prime}\right):=\pi_{s}\left(s^{\prime}\right) \xi\left(s^{\prime}\right) /\left\langle\pi_{s}, \xi\right\rangle$.

Proposition 31. There exists $\xi \in \mathbb{R}_{++}^{S}$ such that $\hat{\delta}_{s}$ is independent of $s \in S$. Moreover, $\xi$ is unique up to scaling, so that $\hat{\delta}$ is unique, and the corresponding measures $\left(\hat{\mu}_{s}\right)_{s \in S}$ are unique up to scaling.

Proof. A representation with a constant discount factor will obtain immediately if we can establish that there exists a vector $\xi \gg \mathbf{0}$ and a number $\hat{\delta}>0$ such that $\hat{\delta} \xi(s)=\delta_{s}\left\langle\pi_{s}, \xi\right\rangle$ for all $s \in S$.

For $S=\{1, \ldots, n\}$, consider the stochastic matrix $\Pi$, whose row $s$ is $\pi_{s}$. Define the diagonal matrix $\Delta$ as follows:

$$
\Delta:=\left[\begin{array}{cccc}
\delta_{1} & 0 & \ldots & 0 \\
0 & \delta_{2} & \ldots & 0 \\
\vdots & \vdots & \ddots & \vdots \\
0 & 0 & \ldots & \delta_{n}
\end{array}\right]
$$

In matrix notation, our problem amounts to finding a $\xi \gg \mathbf{0}$ and $\hat{\delta}>0$ such that $\hat{\delta} \xi=\xi \Delta \Pi$. This amounts to showing that (i) $\xi$ is a (left) eigenvector of the matrix $\Delta \Pi$, and (ii) $\hat{\delta}$ is the corresponding eigenvalue.

We shall say that a matrix is positive if each of its entries is strictly positive. By proposition 29 above, the matrix $\Pi$ is positive, so the matrix $\Delta \Pi$ is also positive. Thus, by the Perron theorem below, such $\xi$ and $\hat{\delta}$ exist, $\xi$ is unique up to scaling, and $\hat{\delta}$ is unique.

By proposition 30, these are the only transformations that we need consider, which establishes that the measures $\left(\hat{\mu}_{s}\right)_{s \in S}$ are unique up to a common scaling.

The Perron Theorem is standard and can be found, for instance, as Theorem 1 in chapter 16 of $\operatorname{Lax}[2007]{ }^{30}$ For completeness, we state the relevant part of the theorem.

TheOrem 5 (Perron). Every positive matrix A has a dominant eigenvalue denoted by $\hat{\delta}$ which has the following properties:

(a) $\hat{\delta}>0$ and the associated eigenvector $\xi \gg \mathbf{0}$.

(30) The Frobenius-Perron theorem generalises the Perron theorem by only requiring that the Markov process be irreducible, ie, for each $s, s^{\prime} \in S$, there exists $n>0$ such that $\Pi^{n}\left(s, s^{\prime}\right)>0$. As discussed in footnote 18, irreducibility of the Markov process corresponds to a weaker version of Uniform Persistence (Axiom X9). 
(b) $\hat{\delta}$ is a simple eigenvalue, and hence has algebraic and geometric multiplicity one.

(c) A has no other eigenvector with nonnegative entries.

Thus, we have the established the existence of the following representation

$$
V\left(f, s^{\prime}\right)=\sum_{s} \Pi\left(s^{\prime}, s\right)\left[\int_{u} \max _{p \in f(s)}[u(p)+\delta V(f, s)] \mathrm{d} \mu_{s}(u)\right]
$$

and

$$
V\left(f, \pi_{0}\right)=\sum_{s} \pi_{0}(s)\left[\int_{U_{K}} \max _{p \in f(s)}[u(p)+\delta V(f, s)] \mathrm{d} \mu_{s}(u)\right]
$$

where $V\left(\cdot, \pi_{0}\right)$ represents $\succsim$.

Proposition 32. Let $\succsim$ have a recursive representation as in (E.6). If $\succsim$ satisfies Aggregate Stationarity (Axiom X6), then $\pi_{0}$ is the unique stationary distribution of the Markov process with transition matrix $\Pi$.

Proof. Recall that $V\left(\{(k, f)\}, \pi_{0}\right)=\sum_{s} \pi_{0}(s)\left[\int u(k)+\delta V(f, s)\right] \mathrm{d} \mu_{s}(u)$. Letting $\kappa:=$ $\sum_{s} \pi_{0}(s) \mu_{s} u(k)$, we see that

$$
\begin{aligned}
& V\left(\{(k, f)\}, \pi_{0}\right)-\kappa \\
& \quad=\delta \sum_{s} \pi_{0}(s)\left[\sum_{s^{\prime}} \Pi\left(s, s^{\prime}\right)\left[\int_{u} \max _{p \in f\left(s^{\prime}\right)}\left[u\left(p_{k}\right)+\delta V\left(p_{h}, s^{\prime}\right)\right] \mathrm{d} \mu_{s^{\prime}}(u)\right]\right] \\
& \quad=\delta \sum_{s^{\prime}}\left[\sum_{s} \pi_{0}(s) \Pi\left(s, s^{\prime}\right)\right]\left[\int_{u} \max _{p \in f_{s}^{x}}\left[u\left(p_{k}\right)+\delta V\left(p_{h}, s^{\prime}\right)\right] \mathrm{d} \mu_{s^{\prime}}(u)\right]
\end{aligned}
$$

By Aggregate Stationarity (Axiom X6), $V\left(\{(k, f)\}, \pi_{0}\right)$ and $V\left(f, \pi_{0}\right)$ represent the same preference. Moreover, by Singleton Indifference to Timing (Axiom X7), they must be affine transformations of each other. Therefore, $\pi_{0}\left(s^{\prime}\right)=\sum_{s} \pi_{0}(s) \Pi\left(s, s^{\prime}\right)$ for all $s^{\prime} \in S$. In other words, $\pi_{0}$ is a stationary distribution of $\Pi$. Since $\Pi$ is positive, the stationary distribution is unique.

Proposition 33. If $\succsim$ has a recursive representation of the form in (E.5) with constant $\delta$, then $\delta \in(0,1)$.

Proof. It follows immediately from the non-triviality of $\succsim$ and from Aggregate Stationarity (Axiom X6) that $\delta>0$. We shall now show that $\delta<1$.

Step 1: Stationarity. As $\pi_{0}$ is the unique stationary distribution of $\Pi$, we have for any $f \in H$, $V\left(f, \pi_{0}\right)=\sum_{s} \pi_{0}(s) V(f, s)$.

Step 2: Constructing Menus. Let $a \in \mathscr{F}_{K}$ be the closed $\varepsilon$-ball around the $p_{k}^{*}$, the uniform lottery over $K$. It is clear that $0<\int_{U} \max _{p \in a} u\left(p_{k}\right) \mathrm{d} \mu_{s}(u)$. But we also have $\int_{U} \max _{p \in a} u\left(p_{k}\right) \mathrm{d} \mu_{s}(u) \leqslant \int_{U} \max _{p \in \mathscr{P}(K)} u\left(p_{k}\right) \mathrm{d} \mu_{s}(u)=\int_{U} \max _{k \in K} u(k) \mathrm{d} \mu_{s}(u) \leqslant$ $\sum_{k \in K} \mu_{s}\left|u_{k}\right|<\infty$, where the last inequality holds because $\mu_{s}$ is nice.

Step 3: $\delta<1$. By the recursive construction of $H$, it follows that there exists a unique act $f^{*}$ that gives the menu $a$ in each period and in every state, ie, $f^{*} \simeq\left(a, f^{*}\right)$. Letting $\eta:=\sum_{s} \pi_{0}(s)\left[\int_{u} \max _{p_{k} \in a} u\left(p_{k}\right) \mathrm{d} \mu_{s}(u)\right]$, we see that $V\left(f^{*}, \pi_{0}\right)=\eta+\delta V\left(f^{*}, \pi_{0}\right)=$ $\eta \sum_{\tau \geqslant 0} \delta^{\tau}$. Since that $\eta>0$ and because $V\left(f^{*}, \pi_{0}\right)$ is finite, we conclude that $\delta<1$, as required. 


\section{E.1.5. Putting it all together}

Proof of Theorem 2. That the representation satisfies all the axioms is straightforward. Consider a preference $\succsim$ that satisfies Axioms X1 X9. By Theorem 4 , $\succsim$ has a finitely additive, EU representation. Separability (Axiom X5 implies, according to lemma 20 and proposition 21. that any such finitely additive EU representation also has a representation based on a regular countably additive measure.

Proposition 25 says that since $\succsim$ satisfies State Contingent Strategic Rationality (Axiom X8), the marginal of $\mu_{s}$ on $\mathfrak{U}_{H}$ is a singleton, and hence by proposition 24, it follows that each $\mu_{s}$ is identified up to a scaling. Propositions 27, 28, and 29 show that Singleton Indifference to Timing (Axiom X7), Uniform Persistence (Axiom X9), and Aggregate Stationarity (Axiom X6) imply the existence a recursive representation. Proposition 31 shows that there exists an equivalent recursive representation with a constant discount factor, and transition probabilities $\Pi$ on $S$, and this representation is unique in the sense of Theorem 2. Proposition 32 shows that because of Aggregate Stationarity (Axiom X6), it must be that $\pi_{0}$ is the unique stationary or invariant distribution of $П$. Proposition 33 shows that $\delta \in(0,1)$ because all utilities from feasible menus are finite. This proves the theorem.

\section{E.2. Proof of Proposition 5}

Let $W \in C(H \times S)$ and consider the function $\Phi W(f, s)$, given by

$$
\Phi W(f, s):=\sum_{s^{\prime} \in S} \Pi\left(s, s^{\prime}\right)\left[\int_{u} \max _{p \in f\left(s^{\prime}\right)}\left[u\left(p_{k}\right)+\delta W\left(p_{h}, s^{\prime}\right)\right] \mathrm{d} \mu_{s^{\prime}}(u)\right]
$$

for all $s \in S$. It is easy to see that $\Phi$ is monotone, ie, $W \leqslant W^{\prime}$ implies $\Phi W \leqslant \Phi W^{\prime}$, and satisfies discounting, ie, $\Phi(W+\rho) \leqslant \Phi W+\delta \rho$ when $\rho \geqslant 0$. If we assume that $\Phi W \in C(H \times S)$ for all $W \in C(H \times S)$, it follows that $\Phi$ is a contraction mapping (with modulus $\delta$ ), and has a unique fixed point, which establishes the proposition. All that remains is to show that $\Phi$ is an operator on $C(H \times S)$.

For each $x \in \mathscr{F}, u \in \mathcal{U}, W \in C(H \times S)$, and $s \in S$, define

$$
\varphi(x, u, s)=\max _{p \in x}\left[u\left(p_{k}\right)+\delta W\left(p_{h}, s\right)\right]
$$

Then,

$$
\begin{aligned}
|\varphi(x, u, s)| & \leqslant \max _{p \in x}\left|u\left(p_{k}\right)+\delta W\left(p_{h}, s\right)\right| \\
& \leqslant\|u\|_{2} \max _{p \in x}\left|\frac{u\left(p_{k}\right)}{\|u\|_{2}}\right|+\max _{p \in x} \delta\left|W\left(p_{h}, s\right)\right| \\
& \leqslant\|u\|_{2} M_{1}+M_{2, s}
\end{aligned}
$$

where $M_{1}:=\max _{x \in \mathscr{F}} \max _{p \in x}\left|\frac{u\left(p_{k}\right)}{\|u\|_{2}}\right|, M_{2, s}>0$, and the bounds follow from the definition of $u \in U$, the compactness of $H$, and the continuity of $W$.

As $W$ is continuous, the function $u\left(p_{k}\right)+\delta W\left(p_{h}, s\right) \in C(K \times H)$ is a continuous, linear functional on $\mathscr{P}(K \times H)$, when the latter is endowed with the topology of weak 
convergence (which is metrisable). Therefore, by the Maximum Theorem, for each $u \in \mathcal{U}$ and $s \in S, \varphi(x, u, s)$ is continuous in $x$.

Before completing the proof, we establish a useful inequality: $\int_{u}\|u\|_{2} \mathrm{~d} \mu_{s}<\infty$ for each $\mu_{s}$. To see that the inequality holds, let us define $W: \mathscr{F}_{K} \rightarrow \mathbb{R}$ as $W(a):=$ $\int_{u} \max _{\alpha \in a} u(\alpha) \mathrm{d} \mu(u)$ for $a \in \mathscr{F}_{K}$. Recall that $p_{k}^{*}$ is the uniform lottery over $K$. Let $a:=\left\{\alpha \in \mathscr{P}(K):\left\|\alpha-p_{k}^{*}\right\|_{2} \leqslant \varepsilon\right.$ for some $\varepsilon>0$ so that $a \in \mathscr{F}_{K}$. Then, for each $u \in \mathcal{U}, \max _{\alpha \in a} u(\alpha)=\varepsilon\|u\|_{2}$. Therefore, $0 \leqslant W(a)=\varepsilon \int_{u}\|u\|_{2} \mathrm{~d} \mu(u)$. But $W((\mathscr{P}(K))=$ $W(K) \leqslant \sum_{k} \mu\left|u_{k}\right|<\infty$ because $\mu$ is nice, which implies that $0 \leqslant W(a)<\infty$.

We will now show that if $\left(f_{n}\right) \in H^{\infty}$ is a sequence that converges to $f \in H$, then $\Phi(W)\left(f_{n}, s^{\prime}\right) \rightarrow \Phi(W)\left(f, s^{\prime}\right)$ whenever $W \in C(H \times S)$, which establishes that $\Phi W \in$ $C(H \times S)$. (Since $S$ is finite, any convergent sequence in $S$ must eventually be constant, which we take to be $s^{\prime}$.)

Consider any sequence $\left(f_{n}\right)$ that converges to $f$. By the bounds established above, $\left|\varphi\left(f_{n}(s), u, s\right)\right| \leqslant\|u\|_{2} M_{1}+M_{2, s}$, and $\|u\|_{2} M_{1}+M_{2, s}$ is $\mu_{s}$-integrable since $\mu_{s}$ is nice (by the inequality established above). Moreover,

$$
\begin{aligned}
\lim _{n \rightarrow \infty} \Phi W\left(f_{n}, s^{\prime}\right) & =\lim _{n \rightarrow \infty} \sum_{s} \Pi\left(s^{\prime}, s\right)\left[\int_{u} \varphi\left(f_{n}(s), u, s\right) \mathrm{d} \mu_{s}(u)\right] \\
& =\sum_{s} \Pi\left(s^{\prime}, s\right)\left[\int_{u} \lim _{n \rightarrow \infty} \varphi\left(f_{n}(s), u, s\right) \mathrm{d} \mu_{s}(u)\right] \\
& =\sum_{s} \Pi\left(s^{\prime}, s\right)\left[\int_{u} \varphi(f(s), u, s) \mathrm{d} \mu_{s}(u)\right] \\
& =\Phi W\left(f, s^{\prime}\right)
\end{aligned}
$$

As $f$ and $\left(f_{n}\right)$ are arbitrary, we conclude that $\Phi W \in C(H \times S)$ whenever $W \in C(H \times S)$. The equalities above rely on the Dominated Convergence Theorem to interchange the order of limits and integration, and the continuity of $\varphi(\cdot, u, s)$ for each $u$ and $s$ to establish the pointwise limit. This completes the proof.

\section{F. Proofs from Section 5}

We present here proofs concerning the behavioral comparison 'greater preference for flexibility'. We begin with some preliminary lemmas. Recall that $L$ is the subdomain of IHCSs.

Lemma 34. Suppose $\succsim^{*}$ has a greater preference for flexibility than $\succsim$ and both $\succsim$ and $\succsim^{*}$ satisfy Independence (Axiom 3). Then, for all $\ell, \ell^{\prime} \in L, \ell \succsim^{*} \ell^{\prime}$ if, and only if, $\ell \succsim \ell^{\prime}$.

Proof. By hypothesis, $\ell \succsim \ell^{\prime}$ implies $\ell \succsim^{*} \ell^{\prime}$, or equivalently, $\ell^{\prime} \succ^{*} \ell$ implies $\ell^{\prime} \succ \ell$. Therefore, it suffices to show that $\ell^{\prime} \sim^{*} \ell$ implies $\ell^{\prime} \sim \ell$. So, let us suppose $\ell^{\prime} \sim^{*} \ell$ and, without loss of generality, assume that $\ell^{\prime} \succ \ell$. Let $\ell^{\dagger} \succ^{*} \ell^{\prime} \sim^{*} \ell-$ by Independence (Axiom 3), it suffices to consider $\ell$ and $\ell^{\prime}$ for which such an $\ell^{\dagger}$ exists - so that for some sufficiently small $\lambda \in(0,1)$, we have $\lambda \ell^{\dagger}+(1-\lambda) \ell \succ^{*} \ell^{\prime}$ but $\ell^{\prime} \succ \lambda \ell^{\dagger}+(1-\lambda) \ell$, which contradicts the hypothesis, thereby completing the proof. 
Lemma 35. Suppose $\succsim^{*}$ has a greater preference for flexibility than $\succsim$, and suppose $\succsim$ and $\succsim^{*}$ have canonical PFC representations $(\mu, \delta)$ and $\left(\mu^{*}, \delta^{*}\right)$ respectively. Then, $\delta=\delta^{*}$, and $V(\ell)=V^{*}(\ell)$ for all $\ell \in L$. Moreover, $V^{*}(x) \geqslant V(x)$ for all $x \in Z$.

Proof. By lemma 34, we know that $\ell \succsim^{*} \ell^{\prime}$ if, and only if, $\ell \succsim \ell^{\prime}$. Thus, $\succsim$ and $\succsim^{*}$ represent the same preference on the restricted domain $L$. Let $V_{L}$ and $V_{L}^{*}$ denote the value functions for the respective canonical PFC representations, restricted to $L$. As $V_{L}$ and $V_{L}^{*}$ represent the same preference, they are affine transformations of each other. Let $\ell^{*} \simeq\left(p_{k}^{*}, \ell^{*}\right)$ denote the IHCS that gives the uniform lottery in each period, so $V_{L}\left(\ell^{*}\right)=V_{L}^{*}\left(\ell^{*}\right)=0$. For each $k \in K, V_{L}\left(\left\{\left(k, \ell^{*}\right)\right\}\right)=\mu u_{k}+0=\mu u_{k}$ and $V_{L}^{*}\left(\left\{\left(k, \ell^{*}\right)\right\}\right)=\mu^{*} u_{k}$. It follows that $\mu u \propto \mu^{*} u$. But $\mu$ and $\mu^{*}$ are canonical measures, ie, $\|\mu u\|_{2}=\left\|\mu^{*} u\right\|_{2}=1$, so it must be that $\mu u=\mu^{*} u$. Hence, $V(\ell)=V^{*}(\ell)$ for all $\ell \in L$, ie, $V_{L}=V_{L}^{*}$.

Now let $\ell_{k} \simeq\left\{\left(k, \ell_{k}\right)\right\}$, which gives us $V\left(\ell_{k}\right)=\mu u_{k}+\delta V\left(\ell_{k}\right)$. Then, $(1-\delta) V\left(\ell_{k}\right)=$ $\mu u_{k}=\mu^{*} u_{k}=\left(1-\delta^{*}\right) V^{*}\left(\ell_{k}\right)$, which implies $\delta=\delta^{*}$, because $\|\mu u\|_{2}=1$ means that $\mu u_{k} \neq 0$ for some $k \in K$.

For any probability measure $\mu$ on $U$ with $\|\mu u\|_{2}=1$, there exist $k, k^{\prime} \in K$ such that $\mu u_{k}>0>\mu u_{k^{\prime}}$. That is, there exist $\ell, \ell^{\prime} \in L$ such that $\ell \succ \ell^{*} \succ \ell^{\prime}$ where $\ell^{*}$ is as above, and hence $V\left(\ell^{*}\right)=0=V^{*}\left(\ell^{*}\right)$. Consequently, for any $x \in Z$, there exists $\lambda \in(0,1)$ and $\ell^{\dagger} \in L$ such that $\lambda x+(1-\lambda) \ell^{*} \sim \ell^{\dagger}$, which means that $\lambda x+(1-\lambda) \ell^{*} \succsim^{*} \ell^{\dagger}$ because $\succsim^{*}$ has a greater preference for flexibility than $\succsim$. This implies $V^{*}\left(\lambda x+(1-\lambda) \ell^{*}\right) \geqslant V^{*}\left(\ell^{\dagger}\right)=$ $V\left(\ell^{\dagger}\right)=V\left(\lambda x+(1-\lambda) \ell^{*}\right)$, from which it follows that $V^{*}(x) \geqslant V(x)$ for all $x \in Z$.

Let $V$ be the value function that corresponds to the canonical PFC representation $(\mu, \delta)$. Notice that since there is no preference for flexibility with respect to continuation problems, for the purpose of assigning utilities, we may restrict attention to the domain $\mathscr{F}\left(\mathscr{P}\left(K \times\left\{z_{\circ}, z^{\circ}\right\}\right)\right)$, where $V\left(z_{\circ}\right) \leqslant V(z) \leqslant V\left(z^{\circ}\right)$ for all $z \in Z$, and $V\left(z_{\circ}\right)<V\left(z^{\circ}\right)$. We now state a preliminary lemma.

Lemma 36. Let $\varphi: \mathcal{U} \rightarrow \mathbb{R}$ be convex and $D \subset \mathcal{U}$ be compact, convex such that $\left.\varphi\right|_{D}$ is Lipschitz. Then, there exists a compact $x \subset$ aff $\mathscr{P}\left(K \times\left\{z_{\circ}, z^{\circ}\right\}\right)$ such that $\max _{p \in x}\left[u\left(p_{k}\right)+\right.$ $\left.\delta V\left(p_{z}\right)\right] \leqslant \varphi(u)$ with equality for all $u \in D$.

Proof. Let $u^{*} \in D$, and let $h_{u^{*}}(u): U \rightarrow \mathbb{R}$ be an affine function such that $h_{u^{*}} \leqslant \varphi$ with equality at $u^{*}$. (The function $h_{u^{*}}$ represents the hyperplane supporting $\varphi$ at $u^{*}$.) Then, there exist $d \in \mathbb{R}^{K}$ and $d^{\prime} \in \mathbb{R}$ such that $h_{u^{*}}(u)=\langle d, u\rangle+d^{\prime}$. We shall now construct the corresponding menu.

Notice that $h_{u^{*}}(u)=\sum_{i} d_{i} u_{i}+d^{\prime}=\sum_{i=1}^{K-1} u_{i}\left(d_{i}-d_{K}\right)+d^{\prime}$ where we have used the fact that $\sum_{i} u_{i}=0$ for all $u \in \mathcal{U}$. Let $\alpha \in$ aff $\mathscr{P}(K)$ be such that $\alpha_{i}=d_{i}-d_{K}$ for $i=1, \ldots, K-1$, and $\alpha_{K}=1-\sum_{i=1}^{K-1} \alpha_{i}$. Also, let $q_{z} \in$ aff $\mathscr{P}\left(\left\{z_{\circ}, z^{\circ}\right\}\right)$ be such that $d^{\prime}=\delta V\left(q_{z}\right)$ (where $V$ has been extended to aff $\mathscr{P}\left(\left\{z_{\circ}, z^{\circ}\right\}\right)$ by linearity and hence uniquely). Now consider $q\left(u^{*}\right)=\left(\alpha, q_{z}\right)$, the signed measure on $K \times\left\{z_{\circ}, z^{\circ}\right\}$ (with marginals $\alpha$ and $\left.q_{z}\right)$. Then, $h_{u^{*}}(u)=u(\alpha)+\delta V\left(q_{z}\right)$.

Recall that $\varphi$ restricted to $D$ is Lipschitz. This implies that the set $x:=\overline{\operatorname{conv}}\{q(u): u \in$ $D\}$ is compact. (Intuitively, the set of 'slopes' and intercepts of the supporting hyperplanes of $\left.\varphi\right|_{D}$ is compact.) It is clear that $x \subset$ aff $\mathscr{P}\left(K \times\left\{z_{\circ}, z^{\circ}\right\}\right)$. For each $q \in x, u\left(q_{k}\right)+\delta V\left(q_{z}\right)$ is an affine function of $u$. Therefore, $\max _{q \in x}\left[u\left(q_{k}\right)+\delta V\left(q_{z}\right)\right]$ is a convex function of $u$. 
By construction, this function is always dominated by $\varphi$ and is equal to $\varphi$ on $D$, which completes the proof.

The proof of Theorem 3 relies on the following Theorem, which characterises dilations.

THeOREM 6. Let $\mu$ and $\mu^{*}$ be probability measures on $\mathcal{u}$. Then, the following are equivalent:

(a) $\mu^{*}$ is a dilation of $\mu$.

(b) $\mu^{*} \varphi \geqslant \mu \varphi$ for every continuous convex function $\varphi: U \rightarrow \mathbb{R}$ that is $\mu+\mu^{*}$ integrable.

Theorem 6 is Theorem 7.2.17 in Torgersen [1991]. It was proved by Blackwell [1953] for the case where the supports of $\mu$ and $\mu^{*}$ are bounded.

Proof of Theorem 3. (a) implies (b). Suppose $\succsim^{*}$ has greater preference for flexibility than $\succsim$. By Theorem 6 , it suffices to show that for every continuous convex function $\varphi: \mathcal{u} \rightarrow \mathbb{R}$ that is $\mu+\mu^{*}$ integrable, we have $\mu^{*} \varphi \geqslant \mu \varphi$.

Fix such a function $\varphi$. We shall show that there exist continuous, convex functions $\varphi_{n}: \mathcal{U} \rightarrow \mathbb{R}$ that are $\mu+\mu^{*}$ integrable, such that (i) $\varphi_{n} \leqslant \varphi_{n+1}$, (ii) $\varphi_{n} \uparrow \varphi$, and (iii) $\mu \varphi_{n} \leqslant \mu^{*} \varphi_{n}$. Since $\varphi_{1}$ is $\left(\mu+\mu^{*}\right)$ integrable, it follows that $\varphi-\varphi_{1}$ is integrable. Therefore, $0 \leqslant \varphi-\varphi_{n} \leqslant \varphi-\varphi_{1}$, so the Dominated Convergence Theorem and (iii) above imply $\mu \varphi \leqslant \mu^{*} \varphi$.

Let $\left(D_{n}\right)$ be an increasing sequence of compact subsets of $\mathcal{U}$ such that $\bigcup_{n} D_{n}$ covers the effective domain of $\varphi$. By standard arguments (because the effective domain has a relative interior in our finite dimensional setting), we may assume that for all $n,\left.\varphi\right|_{D_{n}}$ is Lipschitz. Then, by lemma 36, there exists $x_{n} \subset$ aff $\mathscr{P}\left(K \times\left\{z_{\circ}, z^{\circ}\right\}\right)$ so that $\varphi_{n}(u):=$ $\max _{q \in x_{n}}\left[u\left(q_{k}\right)+\delta V\left(q_{z}\right)\right]$ satisfies $\varphi_{n} \leqslant \varphi$, and $\left.\varphi_{n}\right|_{D_{n}}=\left.\varphi\right|_{D_{n}}$. It follows immediately from the construction (in lemma 36 ) that $\varphi_{n} \leqslant \varphi_{n+1}$ (since $x_{n} \subset x_{n+1}$ ). All that remains is to show that for all $n, \mu \varphi_{n} \leqslant \mu^{*} \varphi_{n}$.

Let $W$ and $W^{*}$ represent the restrictions of $V$ and $V^{*}$ to $\mathscr{F}\left(\mathscr{P}\left(K \times\left\{z_{\circ}, z^{\circ}\right\}\right)\right)$. Thus, for any $x \in Z$, there exists $x^{\prime} \in \mathscr{F}\left(\mathscr{P}\left(K \times\left\{z_{0}, z^{\circ}\right\}\right)\right)$ such that $V(x)=W\left(x^{\prime}\right)$. Moreover, two PFC representations differ if, and only if, they differ on the domain $\mathscr{F}\left(\mathscr{P}\left(K \times\left\{z_{\circ}, z^{\circ}\right\}\right)\right)$. Abusing notation again, let $W$ and $W^{*}$ denote the respective extensions to all compact subsets of $\mathscr{P}\left(K \times\left\{z_{0}, z^{\circ}\right\}\right)$.

Recall that by lemma 35, $V^{*}(y) \geqslant V(y)$ for all $y \in Z$ and $V\left(z^{\circ}\right)>0>V\left(z_{\circ}\right)$. Then, by linearity, we must have $W^{*}(x) \geqslant W(x)$ for all compact $x \subset \mathscr{P}\left(K \times\left\{z_{\circ}, z^{\circ}\right\}\right)$. But $W\left(x_{n}\right)=\int_{u} \varphi_{n}(u) \mathrm{d} \mu(u)$ (which implies, in particular, that each $\varphi_{n}$ is integrable, since $x_{n}$ is compact and $\left.\max \left\{\left|W\left(x_{n}\right)\right|,\left|W^{*}\left(x_{n}\right)\right|\right\}<\infty\right)$, so that we have $\mu \varphi_{n} \leqslant \mu^{*} \varphi_{n}$ as required.

(b) implies (a). Consider the operators $\Phi, \Phi^{*}: C(Z) \rightarrow C(Z)$ defined as follows:

$$
\begin{aligned}
\Phi W(x) & :=\int_{u} \max _{p \in x}\left[u\left(p_{k}\right)+\delta W\left(p_{z}\right)\right] \mathrm{d} \mu(u) \text { and } \\
\Phi^{*} W(x) & :=\int_{u} \max _{p \in x}\left[u\left(p_{k}\right)+\delta W\left(p_{z}\right)\right] \mathrm{d} \mu^{*}(u)
\end{aligned}
$$

As observed in the proof of Proposition 5, both $\Phi$ and $\Phi^{*}$ are monotone and also satisfy discounting, and are therefore contractions. For each $x \in Z, \max _{p \in x}\left[u\left(p_{k}\right)+\delta W\left(p_{z}\right)\right]$ is a continuous and convex function of $u$. Therefore, by Theorem 6 , for any $W \in C(Z)$, 
$\Phi W \leqslant \Phi^{*} W$. Let $\Phi^{n}$ and $\Phi^{* n}$ denote the $n$-th iterates of $\Phi$ and $\Phi^{*}$ respectively. We claim that $\Phi^{n} W \leqslant \Phi^{* n} W$ for all $W \in C(Z)$ and for all $n \geqslant 1$. We have already established this for $n=1$. Suppose this is true for $n-1$, ie, $\Phi^{n-1} W \leqslant \Phi^{*(n-1)} W$. Then, $\Phi\left(\Phi^{n-1} W\right) \leqslant$ $\Phi^{*}\left(\Phi^{n-1} W\right) \leqslant \Phi^{*}\left(\Phi^{*(n-1)} W\right)$, ie, $\Phi^{n} W \leqslant \Phi^{* n} W$, as claimed. Finally, let $V$ and $V^{*}$ be the unique fixed points of $\Phi$ and $\Phi^{*}$ respectively, so that $V \leqslant V^{*}$, which completes the proof.

Proof of Proposition 10 It is easy to see, from the arguments in footnote 20 for instance, that $\succsim$ satisfies the appropriate version of Consumption non-triviality (Axiom 9) if, and only if, there exists $s \in S$ such that $\mu_{s} u \neq \mathbf{0}$. Moreover, the recursivity of the representation implies that if $\mu_{s} u \neq \mathbf{0}$, then for all $s^{\prime} \in S, V\left(\cdot, s^{\prime}\right)$ is non-trivial when restricted to consumption streams.

Let $U_{s}$ and $U_{s^{\prime}}$ respectively represent $\succsim_{s}$ and $\succsim_{s^{\prime}}$ over $\mathscr{F}$ where, as before, $U_{s}(x)=$ $\int_{u} \max _{p \in x}\left[u\left(p_{k}\right)+\delta V\left(p_{h}, s\right)\right] \mathrm{d} \mu(u)$ and similarly for $s^{\prime}$. Following the arguments in lemma 35. we see that when restricted to consumption streams, $\left.U_{s}\right|_{M}$ is a positive affine transformation of $\left.U_{s^{\prime}}\right|_{M}$. It is easy to see that the constant term must be zero, so let us suppose $\left.U_{s}\right|_{M}=\left.\lambda U_{s^{\prime}}\right|_{M}$ for some $\lambda>0$. Then, it must be that $\mu$ and $\mu^{*}$ differ by a scaling of $\lambda, \mu u=\lambda \mu^{*} u$, and $V_{M}(\cdot, s)=V_{M}\left(\cdot, s^{\prime}\right)$.

Now consider the SIHCS $m_{t}^{k}$ that delivers in each period, and in every state, $p_{k}^{*}$, the uniform lottery over $K$, except at time $t+1$, where it delivers the prize $k \in K$. Then, $U_{s}\left(m_{t}^{k}\right) / U_{s^{\prime}}\left(m_{t}^{k}\right)=\lambda>0$. Define $\pi_{s}(\cdot):=\Pi(s, \cdot)$. The probability distribution over states $S$ at time $t+1$, conditional on being in state $s$ in date 1 is $\pi_{s} \Pi^{t}$. Therefore, $U_{s}\left(m_{t}^{k}\right)=$ $\delta^{t} \sum_{\tilde{s}} \pi_{s} \Pi^{t}(\tilde{s}) \mu_{\tilde{s}} u_{k}$, which implies that $\lambda=U_{s}\left(m_{t}^{k}\right) / U_{s^{\prime}}\left(m_{t}^{k}\right)=\frac{\left[\sum_{\tilde{s}} \pi_{s} \Pi^{t}(\tilde{s}) \mu_{\tilde{s}} u_{k}\right]}{\left[\sum_{\tilde{s}} \pi_{s^{\prime}} \Pi^{t}(\tilde{s}) \mu_{\tilde{s}} u_{k}\right]}$ (or at least, such a $k \in K$ can be chosen because $\mu_{s} u \neq \mathbf{0}$ for some $s \in S$ ). But $\Pi$ is fully connected and has a unique invariant distribution $\pi_{0}$, which implies $\lim _{t \rightarrow \infty} \pi_{S} \Pi^{t}=\pi_{0}=$ $\lim _{t \rightarrow \infty} \pi_{s^{\prime}} \Pi^{t}$, which means we must have $\lambda=1$, ie, $\left.U_{s}\right|_{M}=\left.U_{s^{\prime}}\right|_{M}$ and, in particular, $\mu_{s} u=\mu_{s^{\prime}} u$.

Consider now, the state $\tilde{s} \in S$, and let $m \in M$ be such that $V(m, \tilde{s}) \neq 0$. Such an $m$ exists because $V(\cdot, \tilde{s})$ is non-trivial over consumption streams. Let $m^{*}$ be the SIHCS that gives the uniform lottery $p_{k}^{*}$ in each state and in every period. Now, consider the consumption stream $m^{\dagger}$ that gives the consumption stream $\left(p_{k}^{*}, m\right)$ in state $\tilde{s}$ and the SIHCS $m^{*}$ in every other state. Then, $V\left(m^{\dagger}, s\right)=\Pi(s, \tilde{s}) V(m, \tilde{s})=\Pi\left(s^{\prime}, \tilde{s}\right) V(m, \tilde{s})=V\left(m^{\dagger}, s^{\prime}\right)$, which implies $\Pi(s, \tilde{s})=\Pi\left(s^{\prime}, \tilde{s}\right)$ because $V(m, \tilde{s}) \neq 0$.

Although $\left\|\mu_{s} u\right\|$ and $\left\|\mu_{s^{\prime}} u\right\|$ need not be equal to 1 , all that matters in using the arguments in the proof of Theorem 3 is that $\mu_{s} u=\mu_{s^{\prime}} u$. Therefore, we can adapt the arguments from the proof of Theorem 3 and show that (a) is equivalent to (b), which proves the proposition.

\section{References}

D. Ahn and T. Sarver. Preference for flexibility and random choice. Econometrica, forthcoming 2012. 5 
C. D. Aliprantis and K. C. Border (1999). Infinite Dimensional Analysis: A Hitchhiker's Guide, 2nd ed, Springer, New York. 23, 24, 26, 31

F. J. Anscombe and R. J. Aumann. A definition of subjective probability. Annals of Mathematical Statistics, 34:199-205, 1963. 5, 13, 15

G. Bekaert, E. Engstrom, and S. Grenadier. Stock and bond returns with moody investors. Journal of Empirical Finance, 17(5):867-894, 2010. 6

D. Blackwell. Equivalent comparison of experiments. Annals of Mathematical Statistics, 24:265-272, 1953. 42

T. Bollerslev, M. Gibson, and H. Zhou. Dynamic estimation of volatility risk premia and investor risk aversion from option-implied and realized volatilities. Journal of Econometrics, 160:235-245, 2011. 6

J. Campbell and J. Cochrane. By force of habit: A consumption-based explanation of aggregate stock market behavior. The Journal of Political Economy, 107(2):205-251, 1999. 6

E. Dekel, B. L. Lipman, and A. Rustichini. Representing preferences with a unique subjective state space. Econometrica, 69(4):891-934, 2001. 2

E. Dekel, B. L. Lipman, A. Rustichini, and T. Sarver. Representing preferences with a unique subjective state space: A corrigendum. Econometrica, 75(2):591-600, 2007. 2

D. Dillenberger and P. Sadowski. Subjective Learning, working paper, 2012. 5

P. Fishburn. Utility Theory for Decision Making. John Wiley and Sons, New York, NY, 1970. 25

S. M. Goldman. Flexibility and the demand for money, Journal of Economic Theory, 9:203222, 1974. 5

F. Gul and W. Pesendorfer. Self-control and the theory of consumption, Econometrica, 72(1):119-158, 2004. 2

F. Gul and W. Pesendorfer. Random expected utility. Econometrica, 74(1):121-146, 2006. 5

I. Hendel and A. Nevo. Measuring the implications of sales and consumer inventory behavior. Econometrica, 74(6):1637-1673, 2006. 5, 10

Y. Higashi, K. Hyogo, and N. Takeoka. Subjective random discounting and intertemporal choice. Journal of Economic Theory, 144(3):1015-1053, 2009. 4

R. A. Jones and J. M. Ostroy. Flexibility and uncertainty. Review of Economic Studies, 51(1):13-32, 1984. 5

T. C. Koopmans. On the flexibility of future preferences, in Human Judgments and Optimality, ed by M. W. Shelly and G. L. Bryan. New York: John Wiley and Sons, 1964. 5 , 7

D. M. Kreps. A representation theorem for 'preference for flexibility'. Econometrica, 47(3):565-577, 1979. 2, 4, 8

D. M. Kreps. Notes on the Theory of Choice. Westview Press, Boulder, Co, 1988. 25

D. M. Kreps and E. L. Porteus. Temporal resolution of uncertainty and dynamic choice theory. Econometrica, 46(1):185-200, 1978. 7 
R. V. Krishna and P. Sadowski. Preference for Flexibility and the Pricing of Assets. Technical report, Duke University, 2012a. 3, 6, 20

R. V. Krishna and P. Sadowski. Supplement to Dynamic Preference for Flexibility: Unobservable Persistent Taste Shocks. Technical report, Duke University, 2012b. 3, 22, 28

P. D. Lax. Linear Algebra and its Applications. Wiley, New Jersey, 2007. 37

A. Rustichini. Preference for flexibility in infinite horizon problems. Economic Theory, 20:677-702, 2002. 5

P. Sadowski. Conditional preference for flexibility: Eliciting beliefs from behavior. Theoretical Economics, forthcoming 2012. 4,5

P. Schenone. Identifying subjective beliefs in subjective state space models. Technical report, Northwestern University, 2010. 5, 9, 28

D. Smith and R. Whitelaw. Time-varying risk aversion and the risk-return relation. Technical report, New York University, 2009. 6

N. Takeoka. Representation with a unique subjective decision tree. Technical report, Yokohama National University, 2006. 4

N. Takeoka. Subjective probability over a subjective decision tree. Journal of Economic Theory, 136:536-571, 2007. 5

E. Torgersen. Comparison of Statistical Experiments. Cambridge University Press, 1991. 42 